\title{
NBSIR 81-2267
}

\section{Annual Report: Electric and Magnetic Field Measurements}

R. H. McKnight, F. R. Kotter, M. Misakian, and P. Ortiz

Electrosystems Division

U.S. Department of Commerce

National Bureau of Standards

Washington, DC 20234

February 1981

Issued May 1981

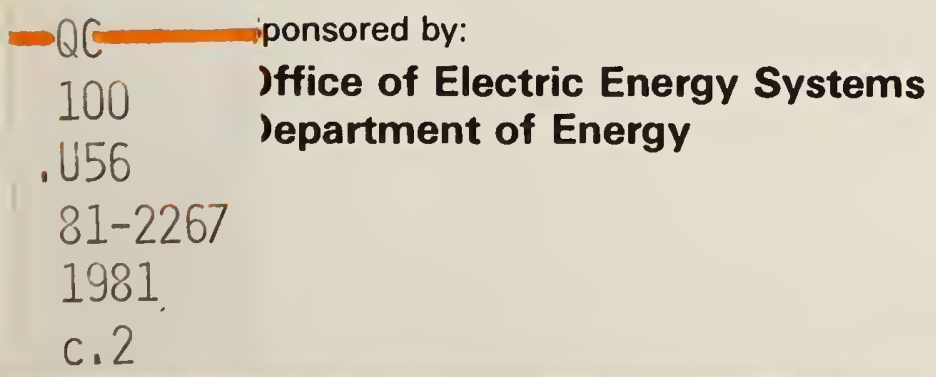



Nas

\section{ANNUAL REPORT: ELECTRIC AND MAGNETIC FIELD MEASUREMENTS}

R. H. McKnight, F. R. Kotter, M. Misakian, and P. Ortiz

Electrosystems Division

U.S. Department of Commerce

National Bureau of Standards

Washington, DC 20234

February 1981

Issued May 1981

Sponsored by:

Office of Electric Energy Systems

Department of Energy

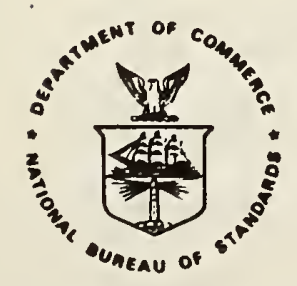

U.S. DEPARTMENT OF COMMERCE, Malcolm Baldrige, Secretary NATIONAL BUREAU OF STANDARDS, Ernest Ambler, Director 


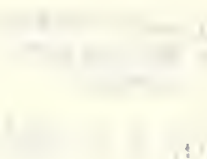


TABLE OF CONTENTS

Page

Summary ....................... i i

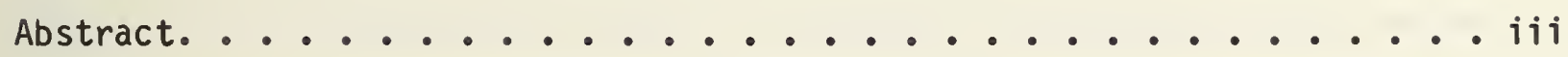

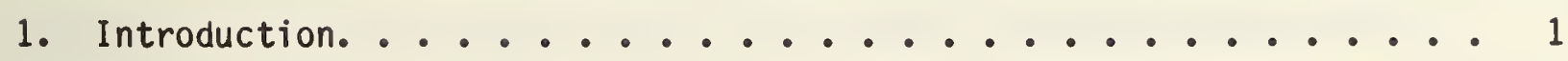

2. Parallel Plate Apparatus...................... 3

3. Errors Associated with Wilson Plate Measurements. ....... 3

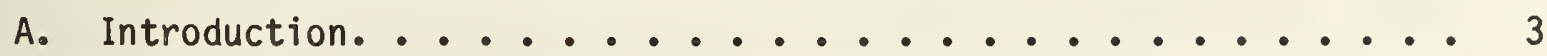

B. Approach ...................... 4

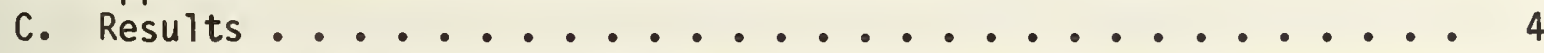

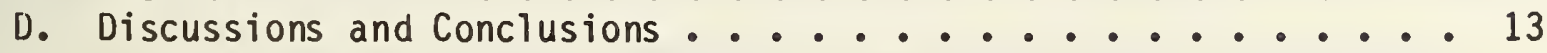

4. Off-Ground Field Measurements ................ 13

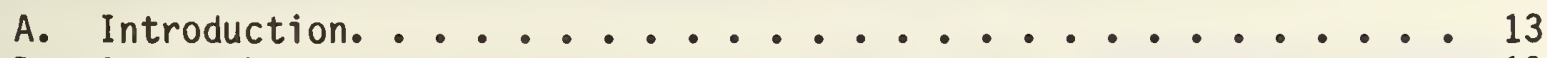

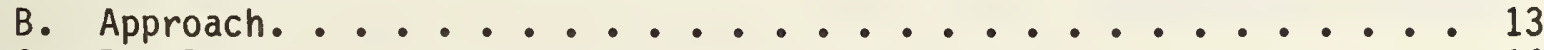

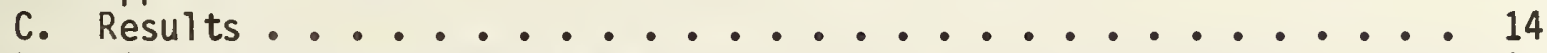

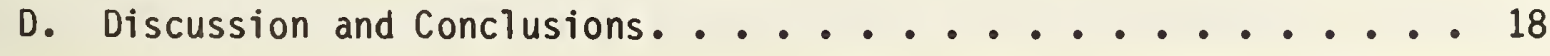

5. Development of a Facility for Producing Space Charge

for Use in Evaluating Ion Measuring Instruments ........ 19

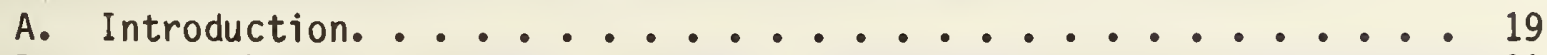

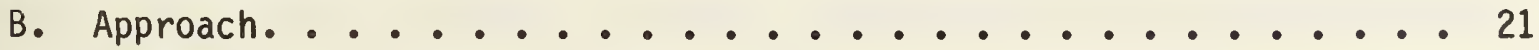

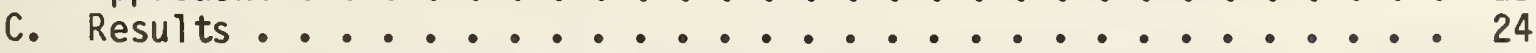

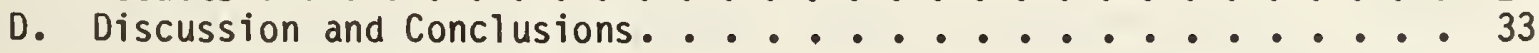

6. Ion Density Measurements. ................... 36

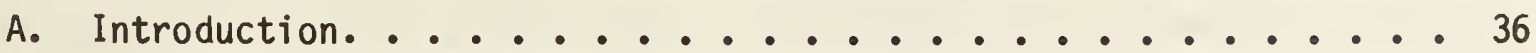

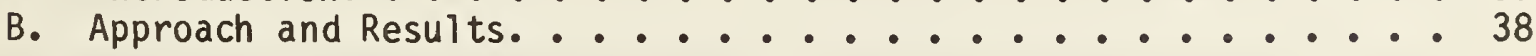

B1. Ion Counters ................. 38

B2. Net Space Charge Measurements. ......... 47

C. Discussion and Conclusions............. 52

7. Discussions and Conclusions ................. 53

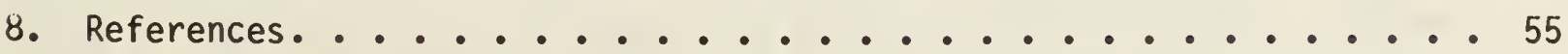

Appendix I - Model Calculations of Perturbations to Ambient

Fields Caused by Instrument Support Structure. . . . . 57 
Summary

The NBS program is concerned with developing methods for evaluating and calibrating instrumentation for use in measuring the electric field and various ion-related electrical quantities in the vicinity of high-voltage direct current (HVDC) transmission lines and in apparatus designed to simulate the transmission line environment.

A parallel plate apparatus designed to produce electric fields in the presence of space charge has been evaluated and used to determine the response of two vibrating plate electric field meters and a field mill to electric fields greater than $\sim 6 \mathrm{kV} / \mathrm{m}$ with attendant current densities up to $\sim 3.5 \mu \mathrm{A} / \mathrm{m}^{2}$. The original one-dimensional model used to predict the operation of the system has been modified to take account of field penetration into the parallel plate region of the apparatus. A field mill, the operation of which is theoretically predicted to be free of significant errors associated with incident current, has been used to verify predictions of the modified theory. The response of the field mill to various field and current density conditions shows that there is no significant error due to incident current. Both vibrating plate meters display errors in indicated field which are a function of the magnitude of the incident current. In this work, both positive and negative fields were used.

Errors associated with lack of coplanarity of Wilson plate sensors with the ground $\mathrm{plane}$ have been investigated using both ac electric fields and dc electric fields with space charge in a parallel plate apparatus. The ac results would be expected to produce enhancement factors which would be the 
same as those for the dc electric fields with space charge if the ions which make up the incident current follow the electric field lines. Substantial errors were observed when the Wilson plate sensors were operated above the ground plane. The magnitude of the errors depended on the sensor geometry and distance above the ground plane with the largest errors being associated with unguarded sensors. Agreement between ac and dc results was good for conditions like those expected under a HVDC transmission line.

Model calculations to estimate errors associated with self-charge of offground field measuring instruments were completed as were estimates of errors contributed by support structures. For symmetrical systems located 1 - $2 \mathrm{~m}$ from the ground plane, errors due to self-charge appear to be small.

A low-speed air-flow facility has been constructed to produce a volume containing space charge which can be used as a source of ions for evaluating and comparing instruments designed to measure various ion related properties. Net space charge densities in the systems can be made temporally smooth and spatially uniform over a given plane in the test volume. Studies have been completed showing the dependence on the net space charge density of air flow, source configuration, and corona-source discharge voltage. In these studies, net space charge density was determined by using a high efficiency particulate air (HEPA) filter, sometimes called an absolute filter.

Studies have been made of small-ion losses at the inlet structure of parallel plate ion counters. Two systems were evaluated; one had substantial 10sses. An assembly in which the geometry was variable was constructed to be used in studies to determine the loss mechanism involved, which appears to be due to fringing fields. 


\section{Abstract}

The NBS program is concerned with developing methods for evaluating and calibrating instrumentation for use in measuring the electric field and various ion-related electrical quantities in the vicinity of high-voltage direct current (HVDC) transmission lines and in apparatus designed to simulate the transmission line environment.

A parallel plate apparatus designed to produce electric fields in the presence of space charge has been evaluated and used to determine the response of two vibrating plate electric field meters and a field mill to electric fields greater than $\sim 6 \mathrm{kV} / \mathrm{m}$ with attendant ion current densities up to $23.5 \mu \mathrm{A} / \mathrm{m}^{2}$. While the field mill appears to be free of significant errors associated with incident ion current, both vibrating plate meters display errors associated with the presence of sufficiently large ion current incident on the sensing element.

Errors associated with lack of coplanarity of Wilson plate sensors with the ground plane have been investigated using both ac electric fields and dc electric fields with space charge in a parallel plate apparatus. Agreement between ac and dc measurements was good for conditions like those expected under a HVDC transmission line.

A low-speed air-flow facility has been constructed to produce a volume containing space charge which can be used as a source of ions for evaluating and comparing instruments designed to measure various ion-related properties. Studies were made of losses at the inlet structure of two commercial parallelplate ion counters.

Model calculations to estimate errors associated with self-charge of offground field measuring instruments indicate errors are small for appropriate geometries . 



\section{Introduction}

The objectives of the investigation are to develop methods for evaluation and calibration of instrumentation which is used in characterizing the electrical environment in the vicinity of high voltage direct current (HVDC) transmission lines and in apparatus designed to simulate the transmission line environment. The motivation for the effort is concern that the electrical environment in the vicinity of high voltage transmission lines, both ac and $d c$, may prove hazardous to living organisms. Suitable instrumentation exists for measurements near ac lines, and there are recommended procedures for making such measurements [1]. In addition to electric fields near dc lines, significant ion concentrations are produced, since the lines are continuously in corona discharge. Electrical parameters of interest near HVDC lines include the electric field, vertical current density, positive and negative charge densities, and net space charge. Related parameters, more difficult to determine, are ion mobilities and species. The production and dispersion of charged aerosols may also be of importance. Measurements of electric field and ion densities are required at or near ground level.

Measurement techniques presently available for determination of these electrical quantities were historically developed to investigate electrical parameters of the atmosphere [2]. The use of these techniques under the conditions existing near HVDC transmission lines requires considerable extension beyond original design considerations. The performance of these devices must be systematically evaluated over the full measurement range expected in the field and, where possible, calibrated using facilities which produce experimentally known or calculable fields and space charge. 
By evaluating the performance of various instruments under different electrical conditions, estimates can be made of errors associated with corresponding measurements. In this way, the optimum operation of the instrument in the laboratory can be determined. Use of the instrument in the field will result in additional uncertainties because of varying climatic conditions and the requirement for long term operation. These types of uncertainties are not addressed in the present study.

The approach to the general measurement problem has been to consider separately the measurement requirements for each of the individual electrical parameters. As a result, the effort described in this report was divided into a number of individual subtasks and is reported as such.

Much of our effort has been directed toward the development of facilities which can be used to simulate certain aspects of the transmission line environment in the laboratory. While the primary function of these facilities is to allow the evaluation of instruments and measurement techniques, their ultimate usefulness in calibration schemes is also of interest. Two facilities have been designed and constructed. One, which produces a dc field with accompanying space charge, has been extensively investigated and used to evaluate the operation of two different types of electric field meters. A second system, designed to produce a region of spatially uniform and temporally smooth space charge, is in a more rudimentary state. Studies done with these facilities and other accomplishments during this year are described in the following sections. 


\section{Parallel Plate Apparatus}

The development of a parallel plate apparatus for generation of dc fields with space charge was completed and used to examine the performance of two types of electric field probes employed for measurements of electric fields near high voltage dc transmission lines. Use of field probes to determine the space charge mobility provided information on the performance of the instrumentation as well as operation of the apparatus. These data indicated that a field mill with phase-sensitive signal detection can function well in the presence of high current densities, but, under the same conditions, field probes with a vibrating-plate design would give erroneous results.

With a valid in-situ determination of mobility, known fields of arbitrary strength, with controllable amounts of space charge, are produced. The data showed that, for a given field strength, the performance of the vibrating-plate probes improved as the current density was reduced. Only examples where the space charge contribution to the total electric field strength is significant were considered. A separate manuscript describing the apparatus and results of the study was prepared and forwarded to DOE in 1980 [3].

\section{Errors Associated with Wil son Plate Measurements}

\section{A. Introduction}

Measurements of vertical currents to ground near a HVDC transmission line are normally made using a Wilson plate, which was invented for use in atmospheric electricity research. In the simplest implementation, the Wilson plate is a conducting sensor, located in or near the ground plane, which is 
connected to ground through a current measuring device. Frequently a guard ring structure surrounds the sensing element. If the Wilson plate is not coplanar with the ground plane, errors in indicated current may arise as a result of the enhancement of the field at the surface of the sensing element.

B. Approach

In an effort to determine errors from lack of coplanarity, initial studies were made with ac electric fields using the parallel plate apparatus briefly discussed in Section 2. These measurements determined the field enhancement factors associated with a series of small square Wilson plate assemblies.

For the situation where there are ions and a nearly uniform dc field, these enhancement factors would be expected to apply if the ions are precipitated to ground along field lines. Some preliminary measurements using the parallel plate apparatus to generate an electric field with space charge were made which confirm this thesis.

C. Results

Eight small square "Wilson plates" were constructed from $0.157 \mathrm{~cm}$ thick copper-clad fiber glass sheet. On six of them a central section of the copper was isolated from an outer ring by a narrow slot (about $0.5 \mathrm{~mm}$ wide) milled through the copper as indicated in Figure 1. The nominal dimensions were as tabulated in the figure. Metal spacers, $7.5 \times 7.5 \times 0.160 \mathrm{~cm}$, were used to elevate the plate above the ground plane. The "in-ground-plane" value was obtained by plotting the current readings as a function of elevation above the ground plane and extrapolating to zero elevation. Using $4 \mathrm{kV}, 60-\mathrm{Hz}$ excitation of the parallel-plate structure with a plate separation of approximately 

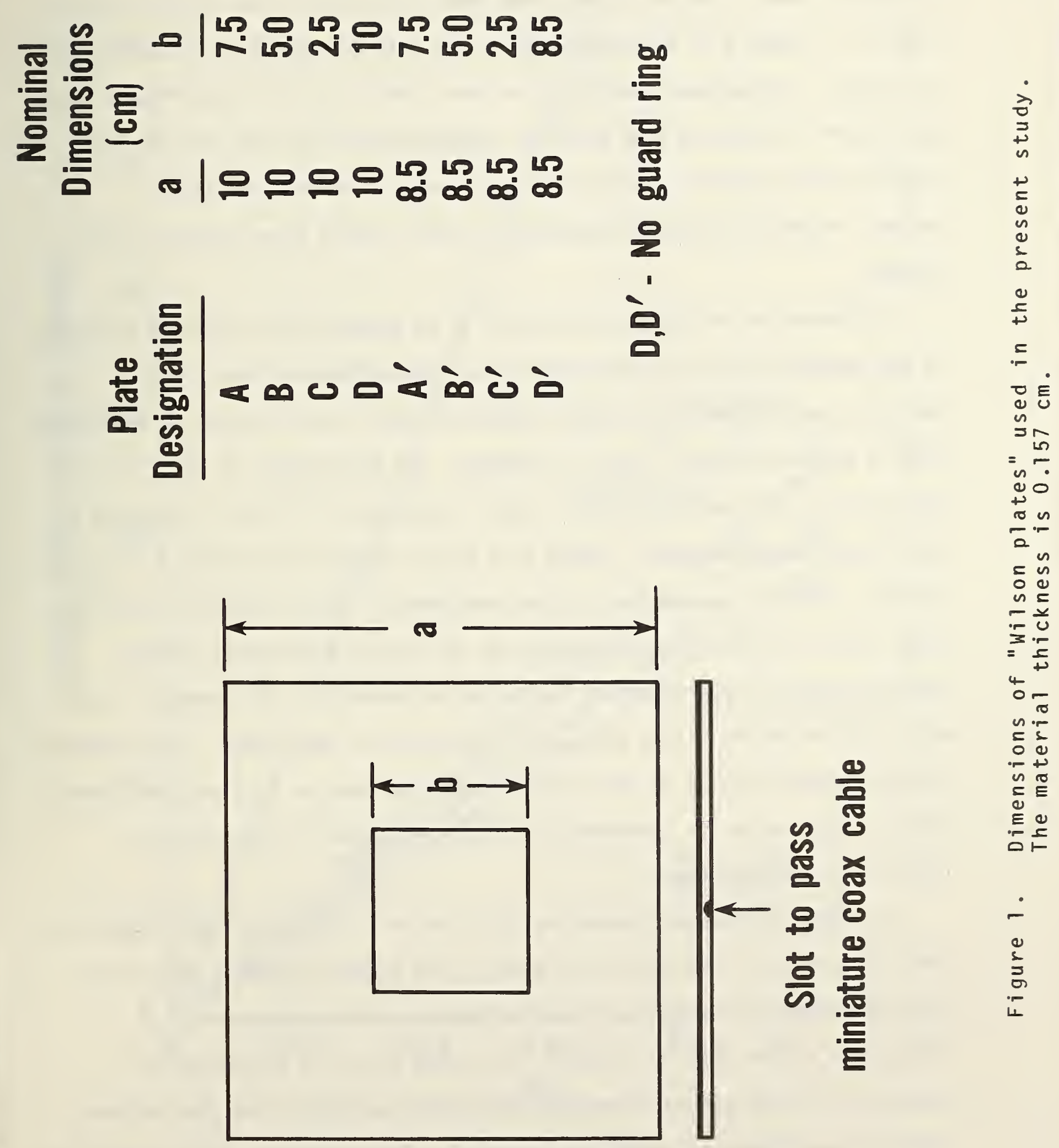
0.5 meter, several series of data were taken with each of the plates listed in Figure 1. Figure 2 is representative of the data obtained with one set $(A-D)$ of plates. The maximum uncertainty in these results is less than five percent, due in part to the fact that with the separation used for the parallel plate facility, the perturbing effects of nearby objects are not negligible. However, relative values are considered correct within a few tenths of one percent.

A theoretical estimate of the error to be expected from a Wilson plate not in the ground plane can be obtained from a consideration of the field perturbation produced by an oblate spheroidal boss on the surface of a plane on which a uniform electric field is incident. The enhancement of the field at the center of the surface of such a boss is tabulated in [4] as a function of the ellipsoidal parameters. Figure 3 is a plot of the values given in [4], and Figure 4 provides a comparison of the experimental results obtained using the small Wilson plates with calculations for two rather arbitrarily chosen spheroids used as approximations to the actual geometry. The geometry of the boss relative to the Wilson plate is as indicated in the figure. These results are considered of value in that they provide confirmation that the experimental results obtained are in agreement with those which can be expected from theoretical considerations.

The value of a guard ring in reducing the error resulting from a departure from coplanarity is indicated in Figure 2. The results obtained with all six plates are shown in normalized form in Figure 5 , which might be used in designing a wilson plate as follows. The value of $a / d$ is determined by $a$ decision as to the minimum elevation above the ground plane and the maximum lateral plate dimensions which are practical. The guard ring width required to 


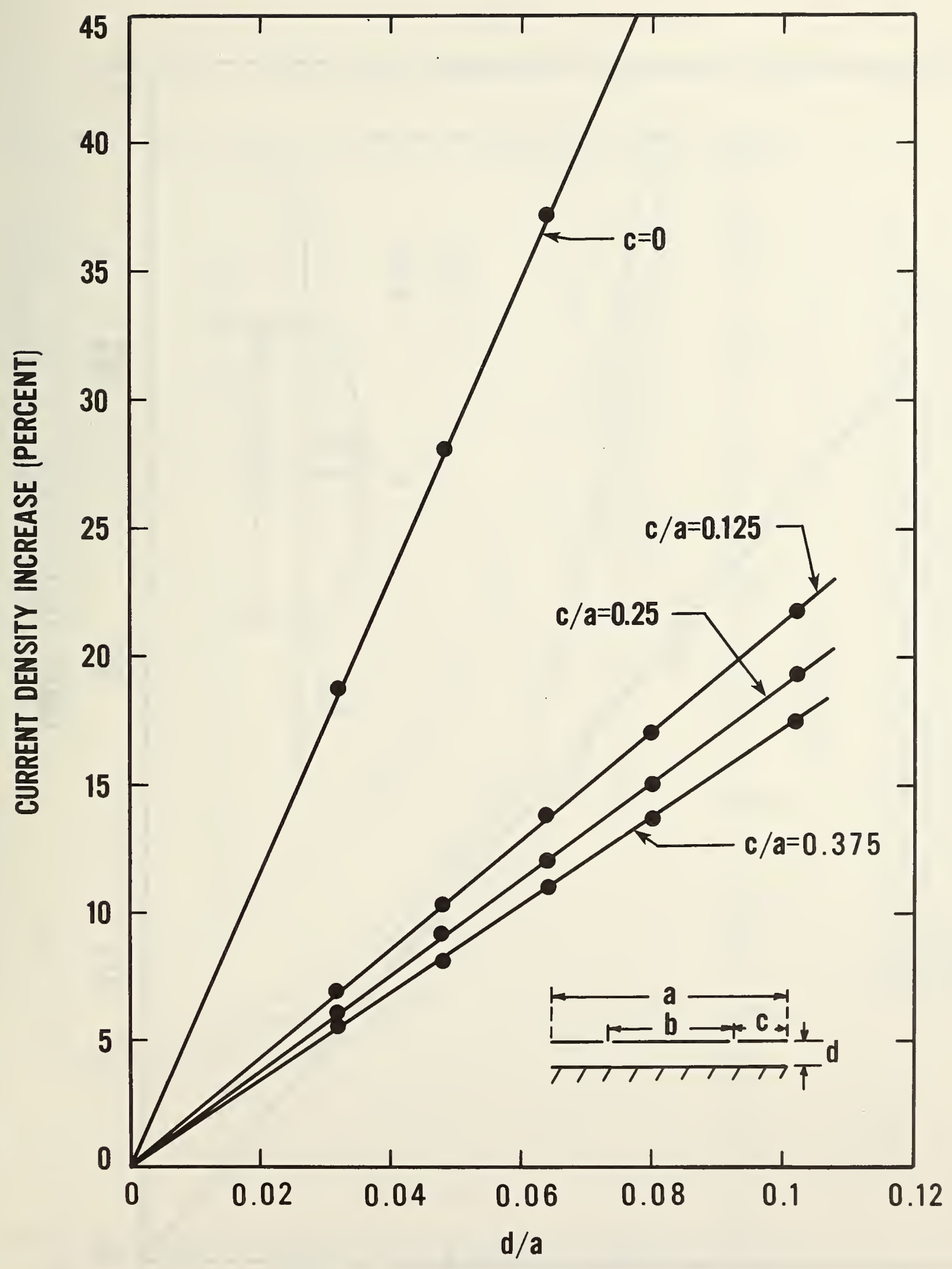

Figure 2. Percentage current density increase for plates $A-D$ (fig. 1). Straight lines have been drawn through
data points to indicate related data. 


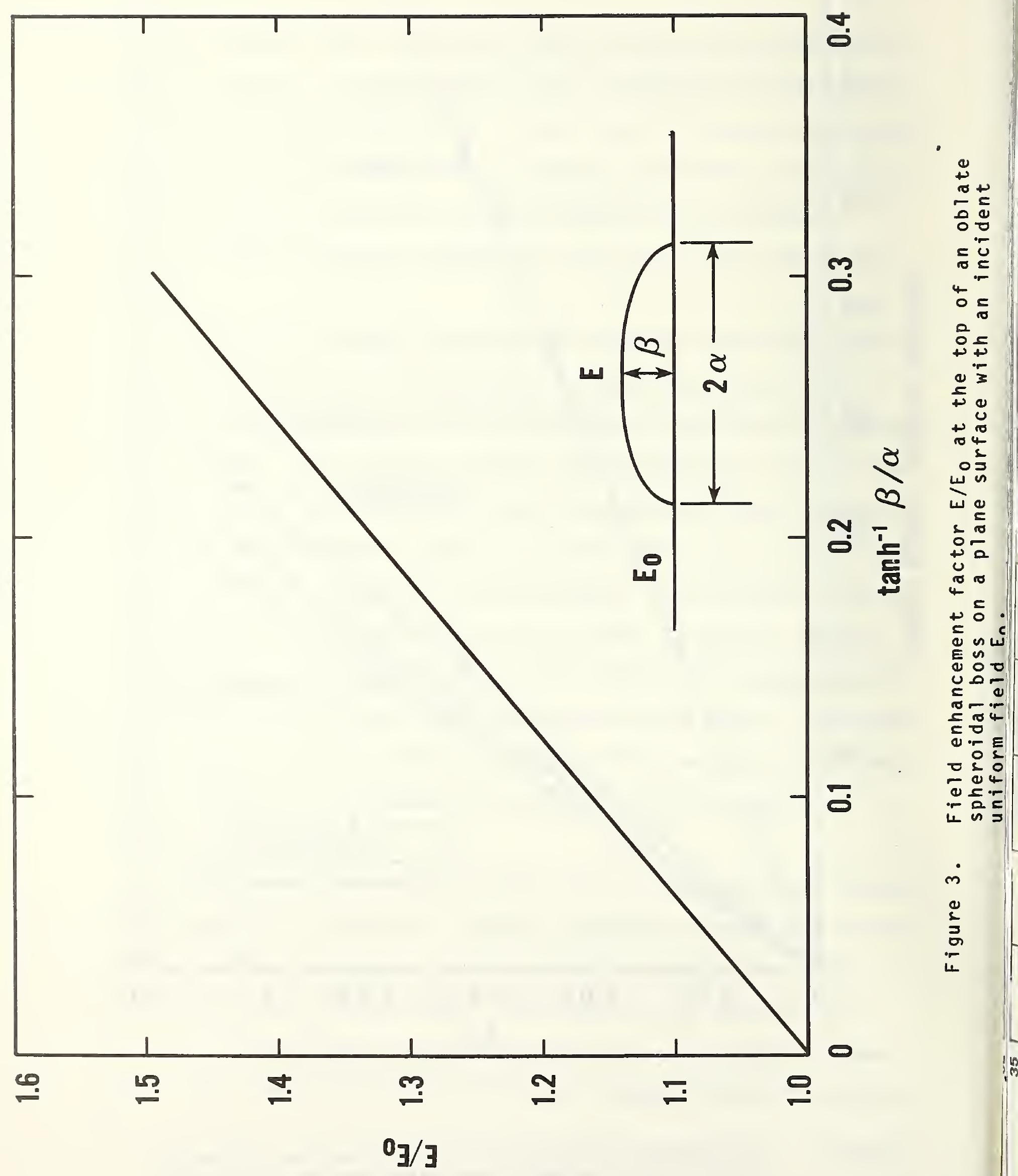




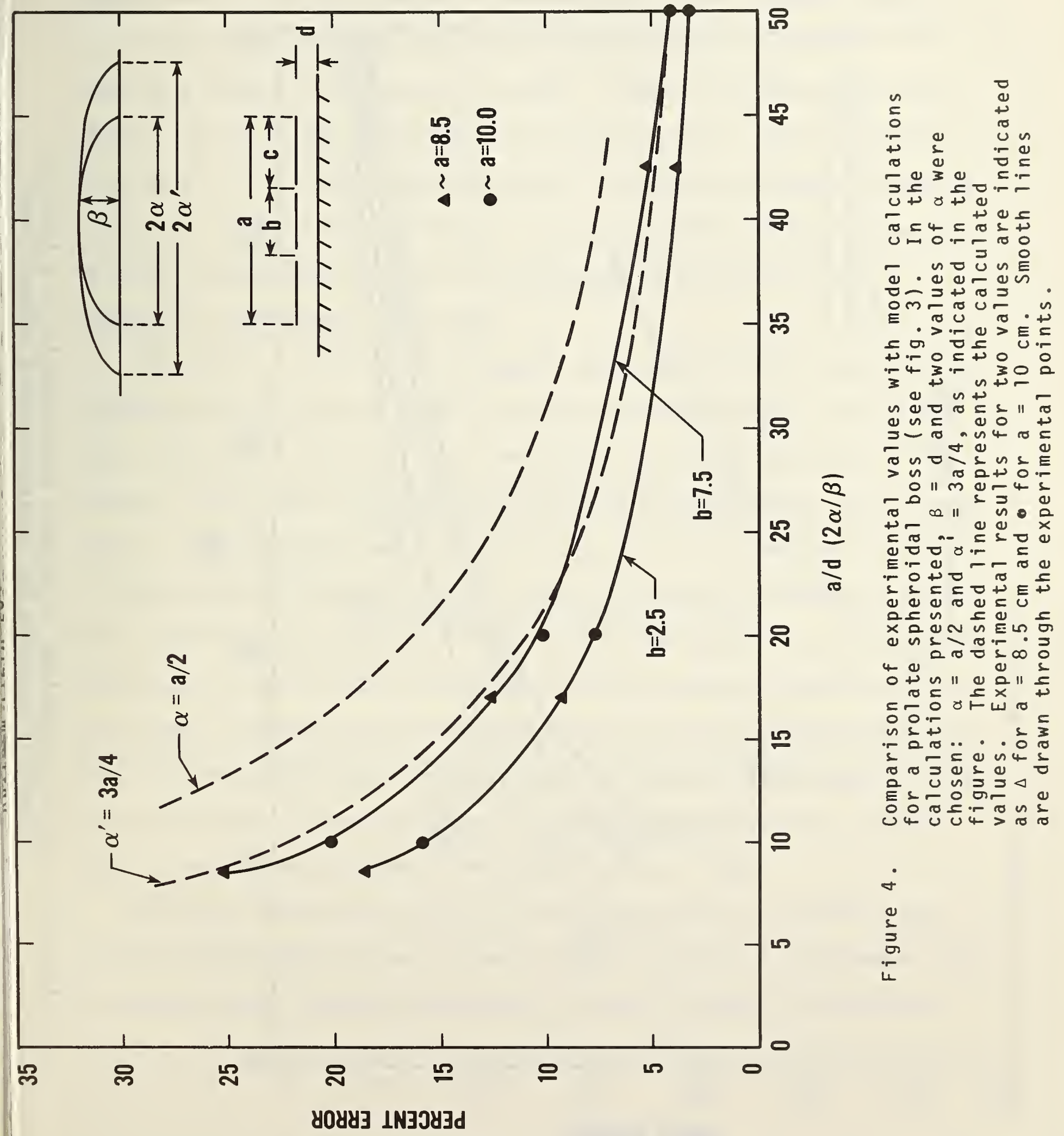




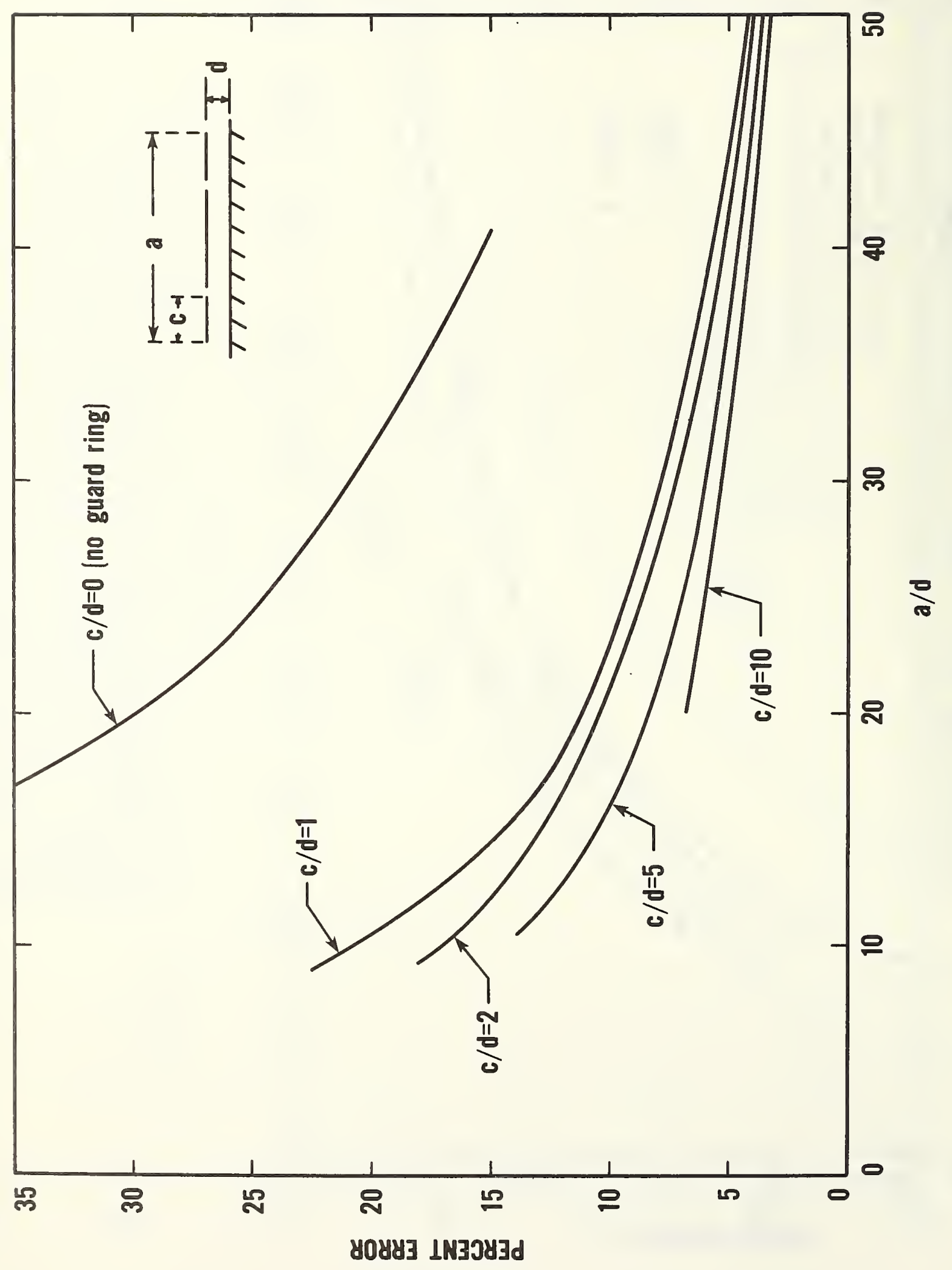

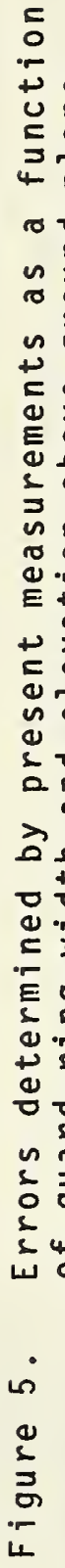


reduce the error resulting from the non-zero value of $d$ to a value considered acceptable can then be estimated by interpolation between the curves given.

It should be noted that the resuits obtained to date are for the capacitive current in an ion-free ac system. Except in the case of the plate with no guard ring (see below) they are expected to apply to the ion current in a dc system if ion motion occurs along the field lines, an assumption expected to be valid in the absence of winds. This is equivalent to assuming that the current to the sensing element will be increased in proportion to the geometrical enhancement of the field.

The effect of fringing at the edge of the unguarded Wilson plate will be different for the ac and dc cases as can be seen from Figure 6. Field lines which terminate on the underside of the sensing plate will contribute to the induced current (the ac case) but ions which traverse these field lines (the dc case) are prevented from reaching the sensing plate by the insulating support. If the resistivity of the insulation is sufficiently high, presumably a charge will accumulate on the surface of the insulation and distort the field. If the resistivity is not so high as to permit significant charge accumulation, the ion current associated with this fringing field will divide between the sensing plate and the ground plane. In either case the results for the unguarded plate with ac (the $c / d=0$ curve in Figure 5) cannot be expected to apply when the plate is used for measurement of ion current.

While only preliminary results of the dc experiment are available, they indicate significantly better agreement with corresponding ac measurements when the sensing plate is guarded than when it is not. The $c / d=0 d c$ values for 


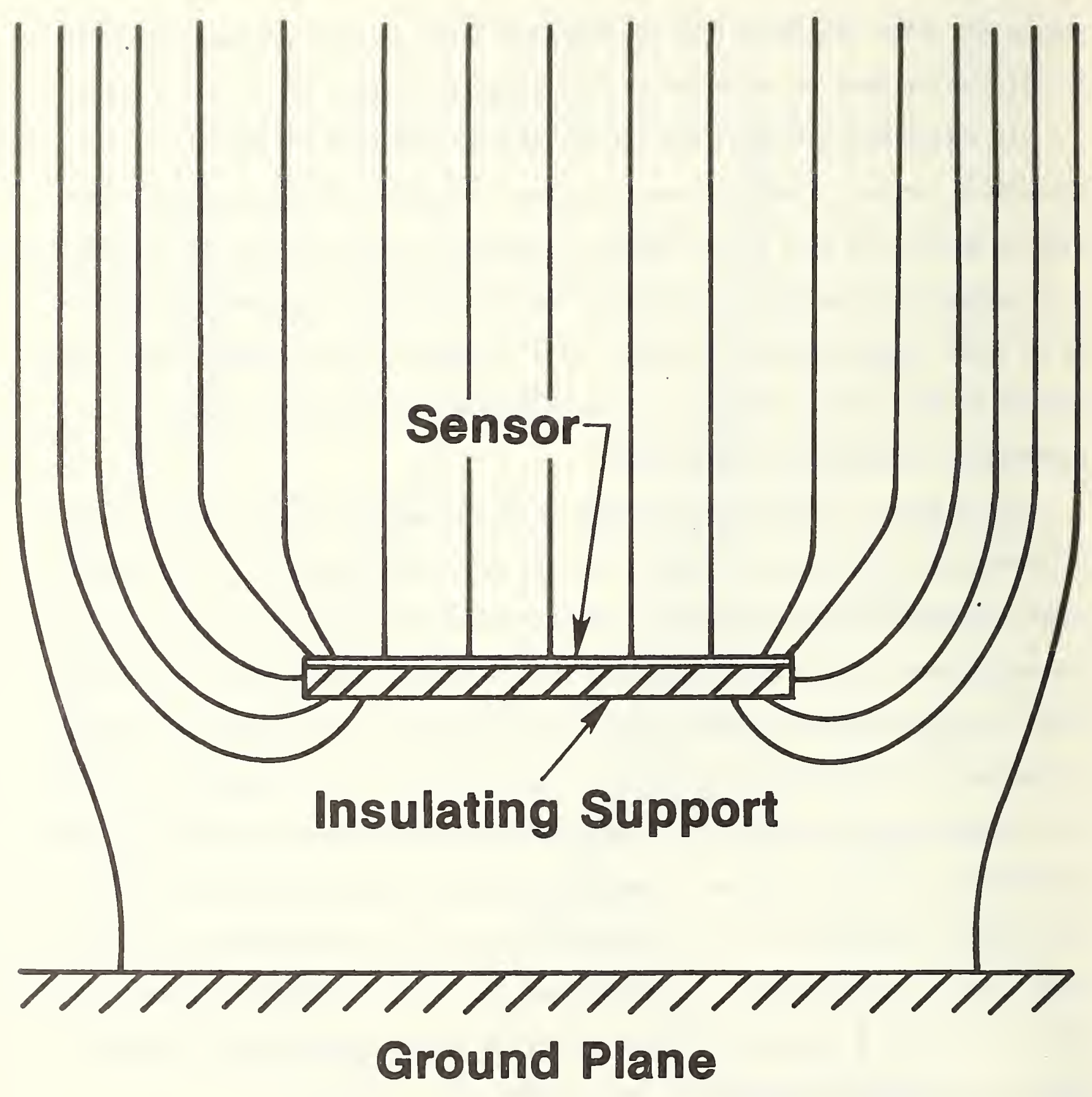

Figure 6. Schematic of flux lines terminating on a sensor plate located above the ground plane. 
enhancement fall below the $c / d=0$ curve of Figure 5 by about 10 percent for values of $a / d$ in the neighborhood of 12 .

D. Discussions and Conclusions

Field enhancement factors for eight different wilson plates raised above the ground plane have been determined using ac techniques. These enhancement factors, when extended to vertical current density measurements, indicate that substantial errors may result if guard rings are not utilized and if the plates are operated above the ground plane. A limited number of preliminary measurements made using a parallel plate apparatus to generate a dc field accompanied by space charge show reasonable agreement with the ac results for all but the unguarded Wilson plates.

\section{Off-Ground Field Measurements}

\section{A. Introduction}

Measurements of the electric field at the surface of the ground are accomplished with rotating vane (field-mill-type) or vibrating plate field meters mounted with their sensing surfaces in or near, and parallel to, the ground plane. Measurements at significant distances above the ground plane are complicated by field distortions introduced by the field meter itself, by the structure used to support it, and by the self-charge which will exist on the meter in the event its potential is not precisely the same as the ambient space potential.

\section{B. Approach}

The field enhancement resulting from the introduction of the conducting body (the field meter) into the field may be determined by full scale or model 
experiments in the laboratory using parallel-plate "uniform-field" calibration structure.

Field meters which are claimed to be free from self-charge errors [5] have been developed for use in atmospheric electricity research. Theoretical analysis of the performance of these meters [5] confirms the validity of those claims; however, only when the instrument is in a "free space" environment, i.e., very remote from other conducting or dielectric bodies. The calculations in the following section show how far from a ground plane such a device must be to insure that the error from this cause will not exceed a specified value.

C. Results

Consider a sphere, divided into two hemispheres insulated from each other, rotating about a horizontal axis passing through the center of the sphere in the insulating plane. If an electric field exists normal to the axis of rotation, an ammeter which joins the hemispheres will indicate an al ternating current resulting from flow of the charges induced on the hemispheres by the field.

The signal current, which is a measure of the field, results from the charge separation between the two hemispheres produced by the field $E_{0}$. That charge difference, obtained by integrating the induced surface charge density over the two hemispheres and subtracting (Ref. 4, p. 226), is

$$
q_{10 w e r}-q_{\text {upper }}=6 \pi E_{0} \varepsilon a^{2} .
$$

The sphere itself may be charged. If the sphere is in free space, this self charge will be uniformly distributed over the sphere and will produce no 
error signal. However, if there are other objects present which disturb the uniformity of the charge distribution, the net charge may introduce an error. Consider the case of a grounded plane near the sphere as in Figure 7 . The problem of the charge distribution on a conducting sphere in the vicinity of a conducting plane is treated by Weber [6]. An approximate solution, which appears valid to better than 1 percent if the radius of the sphere is less than 10 times the distance of the sphere center from the plane, gives for the surface charge density distribution (see Figure 7):

$$
\alpha \approx \frac{Q}{4 \pi a^{2}}\left[1-3\left(\frac{a}{2 h}\right)^{2} \cos \theta\right] .
$$

Integration of this equation gives for the division of the total charge between the hemispheres

$$
\begin{aligned}
& q_{\text {upper }}^{\prime}=\frac{Q}{2}\left[1-\frac{3}{2}\left(\frac{a}{2 h}\right)^{2}\right], \\
& q_{\text {Tower }}^{\prime}=\frac{Q}{2}\left[1+\frac{3}{2}\left(\frac{a}{2 h}\right)^{2}\right] \text {, and } \\
& q_{\text {Tower }}^{\prime}-q_{\text {upper }}^{\prime}=\frac{3 Q}{2}\left(\frac{a}{2 h}\right)^{2} .
\end{aligned}
$$

This charge difference constitutes an error signal as the sphere is caused to 


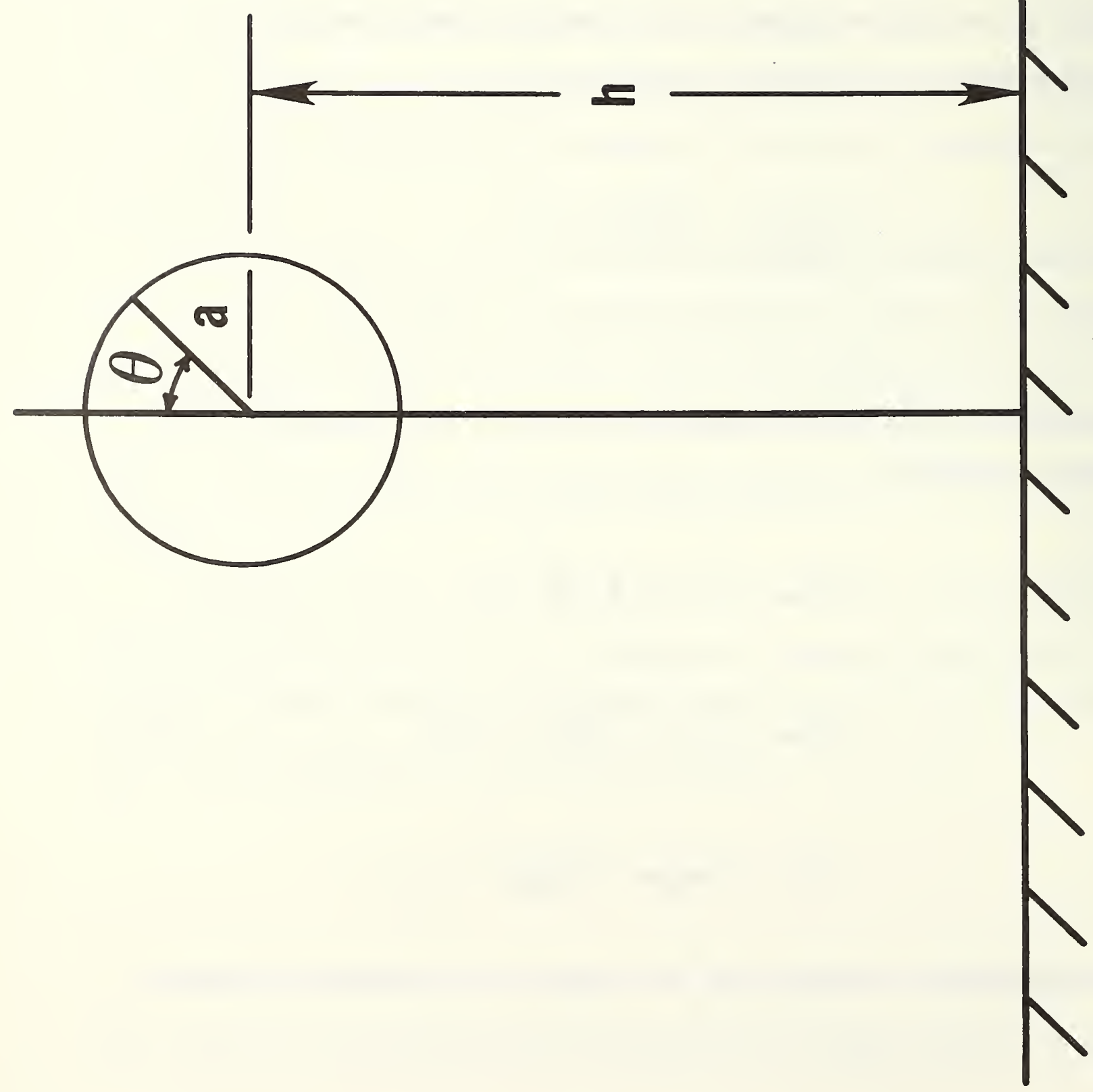

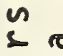

○

<

ब.

45

0

$\leq \stackrel{+}{c}$

$\circ$

$+E$

\&

ज放

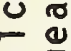

ठठ

D

드

tक

c

응

-

¿ ลे

$\supset 1$

4.

- 4

+

읕 듵

$0=0$

뚱

4

60

舟

र

דים

(U) 눙

E임

용 is

0 的

문 
rotate about a horizontal axis and the current between hemispheres used as a measure of the electric field normal to the axis of rotation.

For the case of a grounded sphere a distance $h$ above a ground plane which bounds a uniform field $E_{0}$, the charge $Q$ is given by $Q=C V$ where $C$ is the capacitance between the sphere and the plane and $V$ is the space potential at the location of the sphere. With the ratio of sphere radius to distance from the ground plane 1:10, the self-capacitance of the sphere is equal to the free-space value, $4 \pi \varepsilon a$, to within 5 percent [7]. Then

$$
Q=4 \pi \varepsilon a E_{0} h
$$

and the error signal is

$$
q_{\text {lower }}^{\prime}-q_{\text {upper }}^{\prime}=\frac{3}{2} \frac{\pi a^{3} \varepsilon E_{0}}{h} \text {. }
$$

The proportional error for this case (obtained by dividing (1) by (5)) is then

$$
\left(q_{1 \text { ower }}-q_{\text {upper }}\right) /\left(q_{1 \text { ower }}^{\prime}-q_{\text {upper }}^{\prime}\right)=\frac{a}{4 h} .
$$


With $a / h \approx 1 / 10$, the error is approximately 2.5 percent. It may be reduced by decreasing the ratio, $a / h$, or by biasing the sphere near the potential of the space adjacent to it.

On the assumption that a field meter of this type could be suspended on a cord or wire between two poles and rotated by means of an internal battery driven motor, with data being recorded on a self-contained battery driven unit, the principal field distortion resulting fram the support structure would be caused by the poles. The question as to how far apart the poles would have to be to reduce the distortion at the location of the meter to an acceptable level has also been explored theoretically (see Appendix I). It has been concluded that pole separations equal to or greater than four times the pole heights will be sufficient to reduce the error from this cause to less than 1 percent.

\section{Discussion and Conclusions}

Model calculations of the errors associated with off-ground electric field measurements made by a rotating, split-sphere field meter indicate negligible error due to self charging effects. An analysis of errors due to attendant support structures for such a meter al so show only a small perturbation of the field at the measuring position. While such an instrument with either spherical or cylindrical geometry would appear to be ideally suited for off-ground field measurements, the errors introduced in the measurement due to ion currents to the device if it were electrically grounded are unknown. A more detailed analysis of the rotating field mill is needed to determine whether its operation either grounded or biased relative to ground is feasible. 
5. Development of a Facility for Producing Space Charge for Use in Evaluating Ion Measuring Instruments.

\section{A. Introduction}

Some of the small ions continually produced by a high voltage dc transmission line are precipitated along field lines to ground near the transmission line. Particulates and aerosols which may be charged by passing near the line have much smaller electrical mobilities than do the small ions and : lay be widely dispersed by ambient winds. The net space charge at a point near a high voltage dc line may be highly variable, presenting a difficult measurement problem. In addition to this, the presence of the electric field produced by the line itself means that the measurements of ion related quantities made above ground level may be influenced.

Clearly, it would be difficult to evaluate basic instrument operation under a transmission line. A less extensive and more controllable system suitable for laboratory investigations is required.

Investigations of instruments designed to measure ion-related properties require a source of space charge which is well characterized, stable, reproducible and controllable. This space charge should be confined to a test volume within which measurements may be made or from which an air sample containing ions may be drawn.

The design philosophy for our effort to develop a standard ion source is based on the requirements listed above. A brief historical description will provide needed background for the present report. Two useful means of producing ionization are the use of corona discharges and radioactive sources. For heal th physics reasons, radioactive sources which are easily handled and 
shielded are used, i.e., alpha, beta, or low energy x-ray sources. Each of these sources produces intense ionization in the region of the source, where positive and negative charges are produced in equal abundance. If ions of only one polarity are required, the source may be biased producing an electric field the direction of which is chosen to inhibit the motion of ions of the unwanted polarity away from the source.

Corona sources differ from radioactive sources in that the source is intrinsically biased so that in the volume surrounding the source, the space charge is predominately of one sign. In addition, these ions are transported away from the source electrode by the electric fields that exist in the space surrounding the source. The number of ions produced in the corona discharge can be controlled to some extent by varying the electrical potential of the source electrode. Either source of ionization may be used to produce ion densities in volumes of a few cubic meters comparable to those found near high vol tage direct current transmission lines.

The second problem to be solved in developing a standard ion source is that of removing the ions produced by corona discharges or radioactive sources from the region of the sources and dispersing them in the test volume. Having accomp 1 ished this, the performance of instruments such as ion counters, Gerdien tubes or net space charge measurement devices may be investigated by either immersing the instruments in the region containing space charge or aspirating ion-laden air from the test volume. The test region, ideally, would have several characteristics - uniform ion density, both spatially and temporally; minimal external electric field penetration; good stability and day-to-day 
reproducibility; variable ion density; and a well characterized ion composition.

The design of the space charge facility should be such to keep the perturbation produced by the measuring instrument to a minimum. In the air flow facility to be described, the volumetric flow rate is much larger than the flow rate through a typical aspiration instrument.

\section{B. Approach}

Initial efforts to produce a standard ion source involved the use of small fans to carry ions away from the region of ionization near an alpha particle or corona discharge source into a closed test volume. Aspiration instruments such as absolute filters or Gerdien conductivity tubes extracted air from this test volume and the exhaust from these instruments was returned to the test volume so as to maintain a closed system. By using several mixing fans, it was felt that through diffusion and air motion within the test volume, a more or less uniform mixture of ions would be achieved. What was observed was an ion distribution which was temporally very uneven. The system could be made fairly reproducible and to some extent spatially uniform but various attempts to remove the temporal fluctuations in the observed ion density were unsuccessful. Several experiments suggested that these fluctuations were related to the lack of smoothness of the air stream carrying ions away from the region of ionization. Because of these results, a different approach was adopted. We decided to concentrate on developing a facility to produce a uniform air stream into which a source of ionization could be placed. The ions could be carried downstream from the source and at some point the ion-air mixture would constitute a "test volume". 
The approach taken was to borrow from the design principles applied to wind tunnel fabrication where low turbulence and uniform air flow are mandatory. A low speed air flow facility was assembled and is illustrated schematically in Fig. 8. The facility is of the open flow type with a fan pulling air through the structure and the return air path through the space surrounding the facility. An inlet structure conditions the air stream by reducing both swirl and turbulence to a low level. The use of flow straighteners and a series of screens to reduce turbulence are standard techniques in wind tunnel design. This first system was not optimized but was intended to serve as a developmental facility to aid in the design of a future sys tem.

An ionization source (in this case, a corona discharge) was located downstream from the turbulence screens. At this point a fairly uniform, low turbulence air stream flows by the wires thus transporting ions into the test section.

Two types of measurements are necessary in investigations of the suitability of this facility for use as a standard ion source. The first involves measurements related to the air stream of such things as the velocity profile and the degree of turbulence. The second is of the uniformity of the ion density produced in the test volume. A hot film anemometer was used for the air flow measurements. For the ion density measurements, an absolute filter assembly was used to measure the ion density at a selected location in the test volume. A sampling tube connected to the inlet of the filter assembly was scanned across the test volume at a selected height above the floor of the flow facility. The current from the filter is proportional to the ion density 


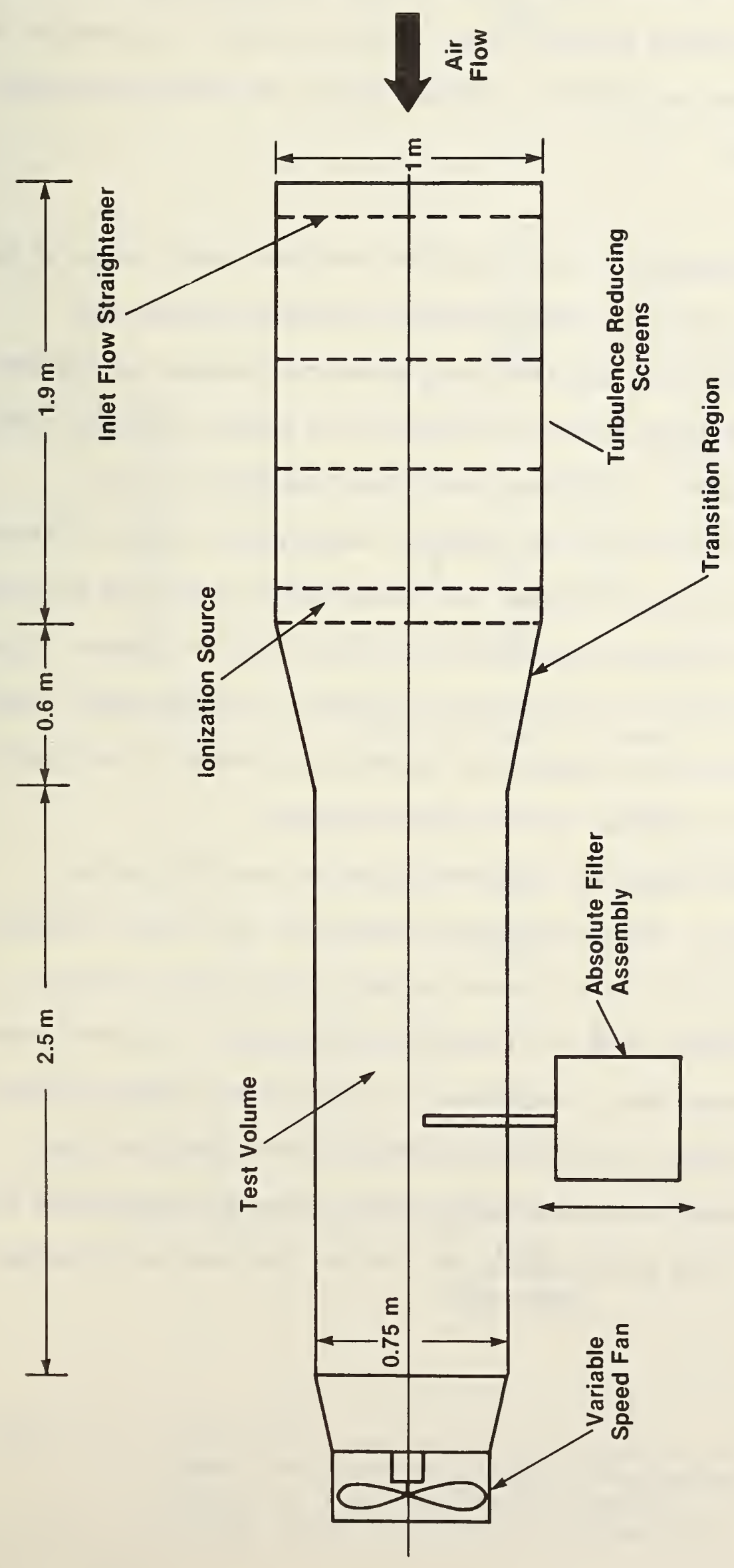

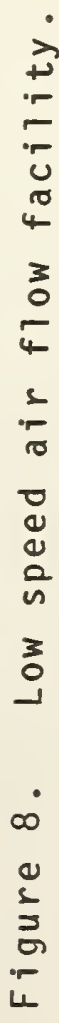


at a given location of the probe. The corona discharge source was designed so that the ground plane and wire configurations could be varied. By studying the ion distributions produced by different configurations, the ionization source design can be optimized.

C. Resuits

Because of the developmental nature and the less than ideal design of the first air flow facility, air flow characteristics far from optimum were expected. Flow characteristics obtained using a hot film anemometer confirmed this expectation. The average speed was uniform to \pm 3 percent over the central two-thirds of the structure. Turbulence levels were substantial over approximately the outer half of the air stream. Measurements made at different locations in the flow facility indicated the turbulence was generated by screen and ion source support structures protruding into the inlet air stream. Also, no attempt was made to design a satisfactory transition section. Since design and construction of a new, more substantial system was planned in the future, no attempts were made to optimize the existing structure.

Initial source development was concentrated on the use of a corona discharge to produce ions. The space charge produced by the corona-discharge ion sources depends primarily on (i) corona voltage, (ii) speed of the air stream flowing by the source, and (iii) source configuration. For the coronadischarge ion source experiments, simple one- and three-wire configurations were used with ground planes represented by arrays of five parallel wires. These configurations as well as a seven-wire source using a screen ground plane are shown in Figure 9. The ground planes for the one and three wire sources 


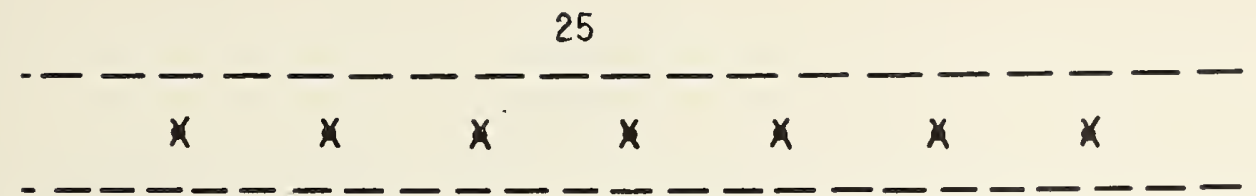

(a)

$x$

(b)

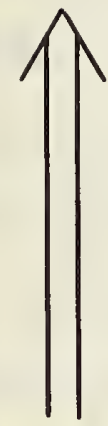

$: \quad \times \quad:$

(c)

\section{Air flow}

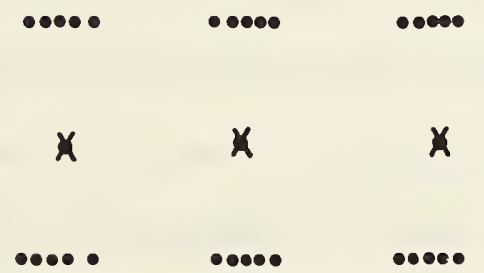

(d)

$\vdots \quad \times \quad \vdots \quad \div \quad \times \quad \vdots$

(e)

$1 \mathrm{~m}$

(Top view)

Figure 9. Corona ion source configurations, drawn to scale. Not shown are support frames, insulators, etc., $x$ - corona wires; -..- - grounded screens; .... - ground wires. The corona wires are $64 \mu \mathrm{m}$ in diameter. 
are much more transparent than source $9 \mathrm{a}$, and represent extremes of electric field orientation relative to air flow.

The screen material was aluminum window screen while the individual ground

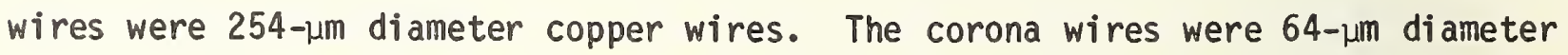
stainless steel. Scans across the test volume using the absolute filter at a fixed distance above the floor of the enclosure for source configuration [a] (Fig. 9) with 7 wires are shown in Fig. 10. Distances above the floor and across the test volume are indicated. The curves, which are proportional to the ion density at a given point in the test volume, are all plotted to the same scale and the error bars represent a \pm 5 percent deviation from the reference value. The fluctuations indicated are representative of the temporal smoothness of the ion density. The fall off in ion density near the right hand side of the volume is an artifact of the scanning technique. A comparison of the ion distribution produced by source configuration $9 a$ with 3 and 7 wires is shown in Fig. 11. The traces shown are for scans across the test volume at 3 different distances above the floor of the flow facility and are plotted to the same scale as in Fig. 10. There is substantial improvement in the uniformity of the ion density for the greater number of source wires.

Despite the turbulence and lack of uniformity observed in the air flow, the ion density is remarkably uniform in the plane scanned. A proposed explanation for this observation is that the uniformity is due to the dispersal and mixing of the space charge by internal Couloumb forces during transit from the source region to the region of observation. The turbulence itself could be expected to be responsible for some mixing. 


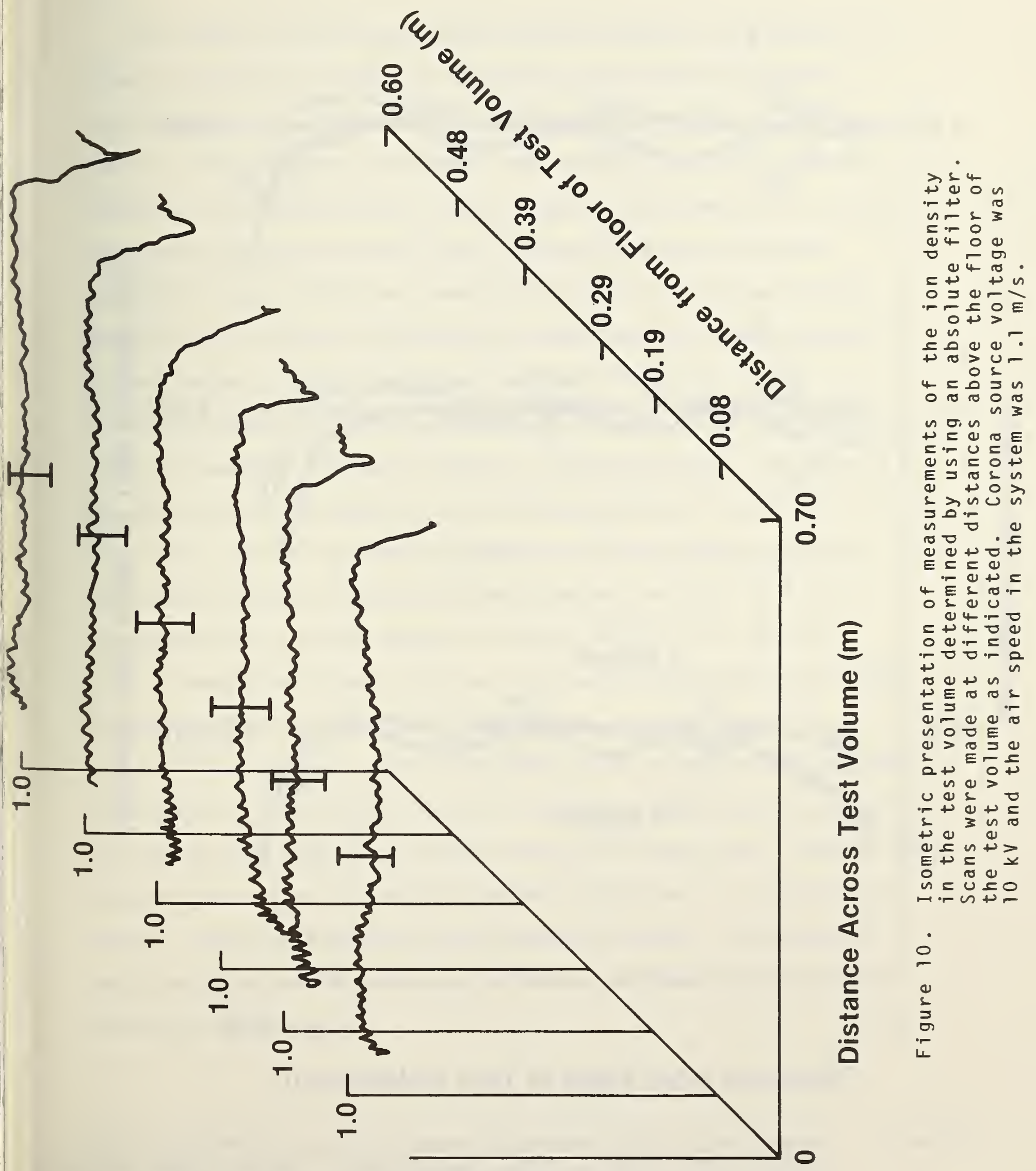

(sł!un 'ןəy) Kł!suәg uol 


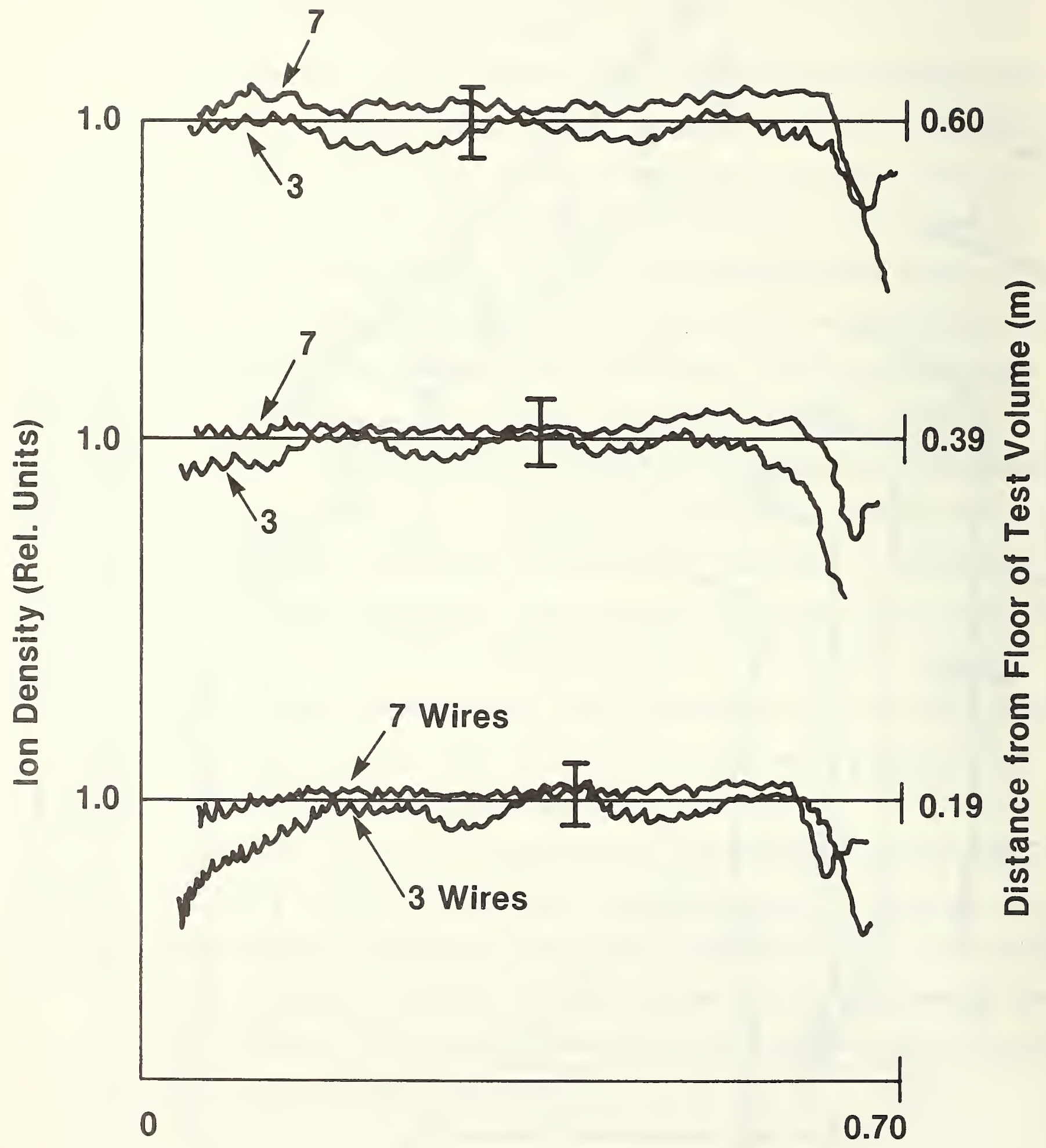

Distance from Edge of Test Volume (m)

Figure 11. Comparison of ion densities produced by two different corona sources with 3 and 7 wires respectively. Scans across the test volume were made at selected distances above the floor of the test volume. For each set of measurements the corona voltage was $10 \mathrm{kV}$ and the air speed $1.1 \mathrm{~m} / \mathrm{s}$. 
For sources b-e, the space charge density, produced as a function of corona voltage and air speed, was determined at the midplane of a given location in the test volume using the absolute filter probe. As expected, the spatial uniformity varied considerably from source to source and even more strongly with increasing corona voltage. Rather than present all of the space charge density profiles obtained, some representative data for source configurations (b) and (d) are summarized in Figure 12. The space charge density shown in Figure 12 represents an average over the middle one third of the test volume. Similar results were obtained for sources $(c)$ and $(e)$, al though for these particular configurations the space charge density profiles were very nonuniform at higher voltages. This nonuniformity, seen above $20 \mathrm{kV}$, may be associated with observed, large-amplitude mechanical oscillations of the source wires and with the onset of negative corona from the ground wires. The space charge produced in these experiments ranged from $3-10 \times 10^{-8} \mathrm{c} / \mathrm{m}^{3}$ (corresponding to a singly charged ion density of 2-6 $\times 10^{11}$ ions $/ \mathrm{m}^{3}$ ), which is comparable to that found near the ground in the vicinity of an HVDC transmission line.

While there appear to be no technical barriers associated with producing large values of space charge density, the problem of reducing the space charge below the minimum generated at corona onset has not been solved. Although the measured space charge density is a function of air flow, it is desirable to develop a system in which this is an independent variable. Therefore, as described below, both electrical and mechanical techniques have been used to control the space charge. 


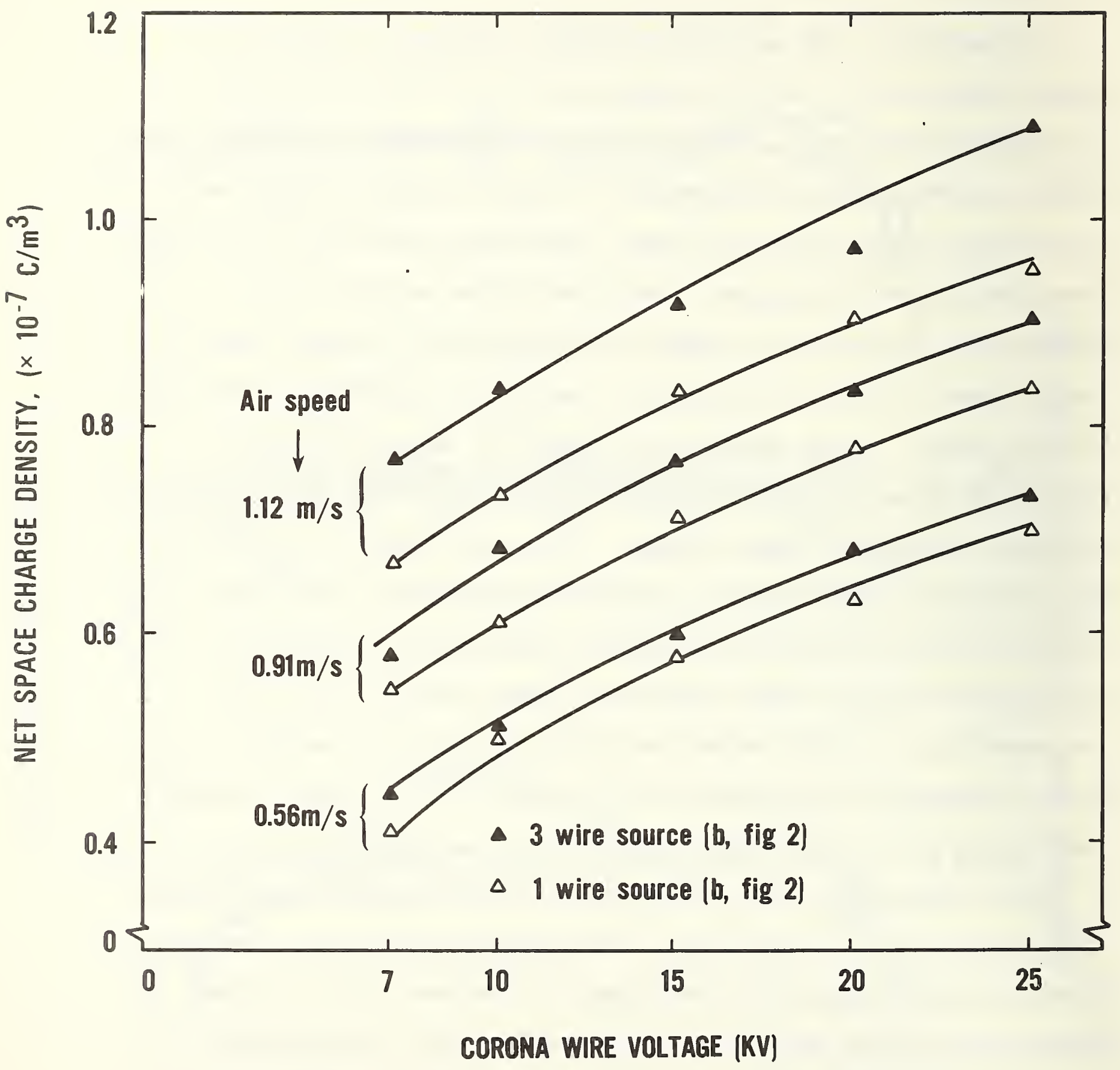

Figure 12. Space charge density produced by 1 wire (source (b), fig. 9) and 3 wire (source (d), fig. 9) source configurations. Air speeds shown are at the source. Errors associated with space charge measurements are estimated to be $\pm 10 \%$. 
Initially, a number of grounded screens (ordinary aluminum window screen mesh) were placed downstream from the ion source. This resulted in an attenuation of approximately 6-8 percent per screen. Since control over orders of magnitude is required, an electrical filter has been constructed. The filter consists of a 3-screen sandwich, in which the center screen can be energized and the outer screens grounded. The attenuation curve obtained for this filter is shown in Figure 13. As previously, the reported space charge density is an average over the central region of the test volume normalized to the initial value. These measurements were made using source (a) (Figure 9) operated at $10 \mathrm{kV}$. There is an initial rapid drop in ion density, but above 300 volts, there is little attenuation with increasing voltage. This is not understood, but is not inconsistent with the hypothesis that a fraction of the space charge is due to charged aerosols or particulates, the motion of which would be dominated by aerodynamic, not electrical, forces for the present conditions.

These charged particles may result from the circulation of room air into the system with subsequent charging of particulates in the corona region, or from charging of particulates in the laboratory. Since the air flow facility is an open return system, this is unavoidable. In a future system, absolute filters will be used at the inlet to insure the air entering the system will be clean and free of charge.

The previous discussion has focused on corona discharge ion sources. In our initial work, however, $\alpha$-particle sources were used to produce ionization. Radioactive sources have been used by others in earlier ion effects research and their employment as sources of space charge in exposure chambers for 


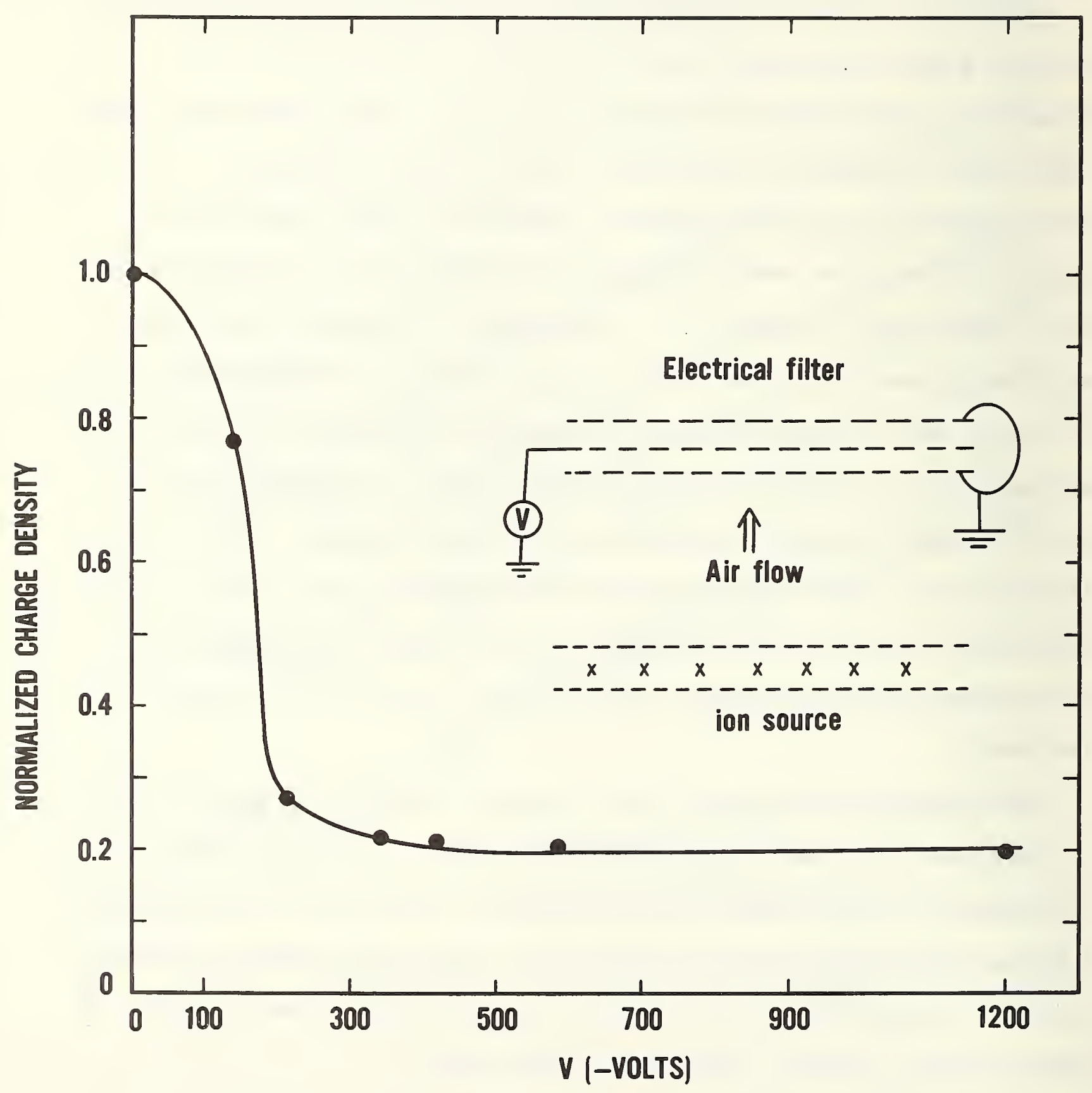

Figure 13. Attenuation of space charge density by an electrical filter. Screen spacing is $0.025 \mathrm{~m}$. The filter-to-source spacing was appoximately $0.15 \mathrm{~m}$. 
biological experiments has been recently suggested, since, in contrast to a corona source, there is no ozone production. In an effort to provide some comparison between space charge produced by $\alpha$-particle and corona discharge ion sources, a limited number of measurements were made using $\alpha$-particle sources. In these measurements, five $\alpha$-particle sources were equally spaced along a vertical wire located at the position of the corona wire in source (b) of Figure 9. The wire was biased to a potential of $+1 \mathrm{kV}$ and space charge density measurements made as a function of source location and air flow using the absolute filter. A comparison between the space charge density produced by the $\alpha$-sources and a single-wire corona source is indicated in Figure 14 . The differences in the dispersal of ions is striking. In other measurements, using more sources with different source-ground plane configurations, very nonuniform distributions were consistently observed.

Further investigations using radioactive sources and the general question of the distribution of ions in the flow facility will be deferred until a new air flow system is constructed.

\section{Discussions and Conclusions}

Much of the work presented above is considered exploratory, since a cursory literature examination revealed little previous work which could be related to the task of producing a volume of space charge with characteristics suitable for use in evaluating ion measuring instruments. The broad dispersal of ions from the various sources studied was not expected. It was found that a single-wire corona source is almost as effective at filling the flow system with charge as is a seven-wire source. The amount of space charge produced is not strongly dependent on total corona current. The speed of the air flowing 


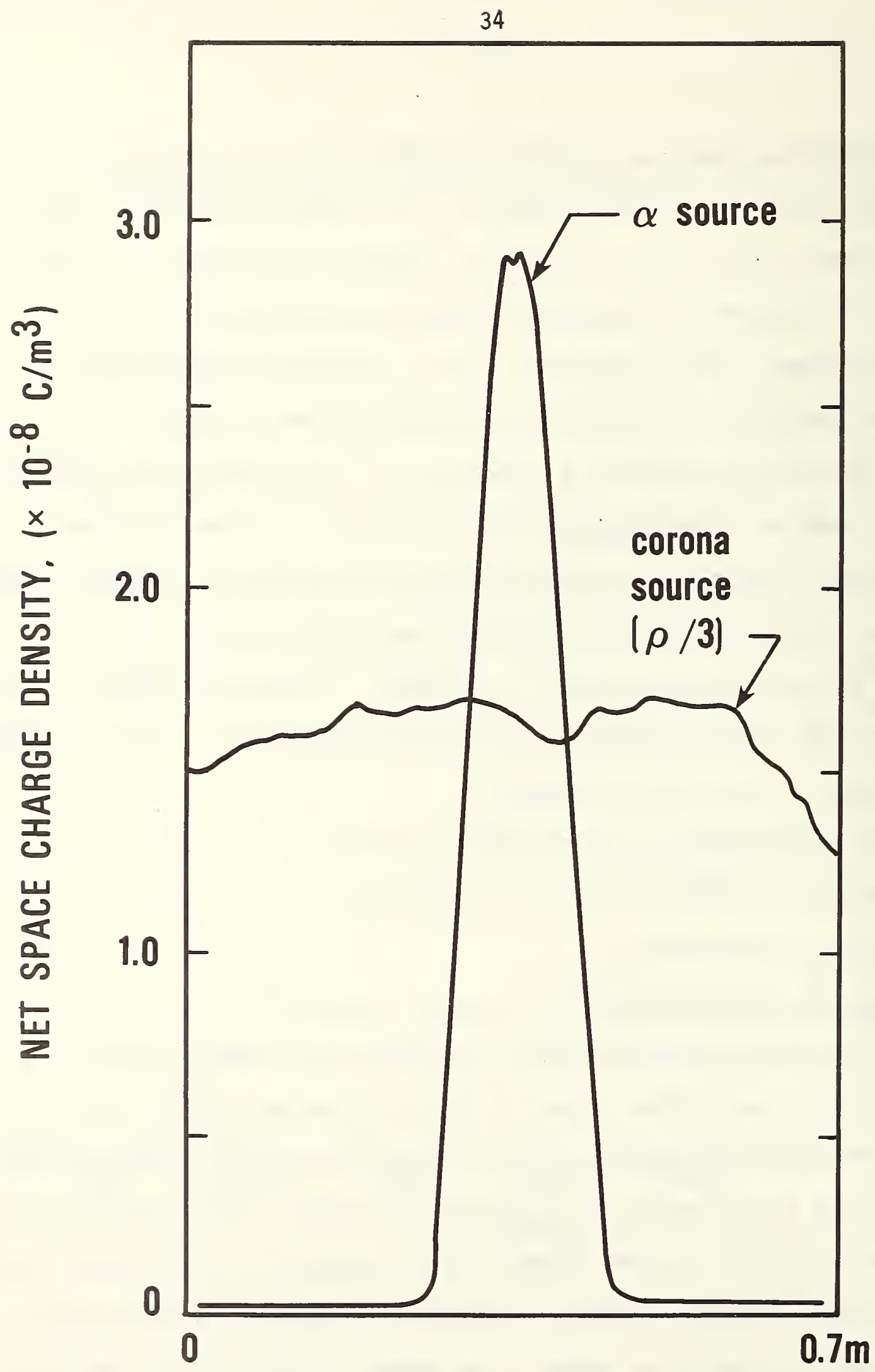

Figure 14. Space charge density produced by an a-particle source (described in the text) maintained at $+1 k V$ bias and by corona source (b) (fig. 9) at $10 \mathrm{kV}$. Air flow was $0.56 \mathrm{~m} / \mathrm{s}$ for both measurements. The space charge produced by the corona source is divided by 3 for presentation. The horizontal axis is the distance from the edge of the test volume. 
across the source region most significantly influences the charge density. More "open" ground planes produce higher ion densities, but care must be taken to insure there is no corona discharge from the ground plane. Based on the results of experience with the developmental flow system, a new flow system has been designed and is being constructed. This new system, which will be described in future communications, incorporates a number of improvements including a more substantial mode of construction, a continuously variable speed fan, a new flow-straightener section with absolute filters, a larger area ratio between the flow-conditioning section and test volume, turbulence screens with frames which do not protrude into the air stream and a new transition section design. Some of these changes will substantially improve the quality of the air flow while others will make the facility more suitable for use in instrunent comparison and calibration.

It has been shown that this approach can produce a volume containing a spatially uniform and temporally smooth space charge, with maximum ion densities equal to or greater than those found under a dc transmission line. For purposes of instrument evaluation, this space charge is made up essentially of ions of one sign, so that the net space charge density and homopolar charge densities are the same.

A number of important questions have been left unanswered and will be addressed in future work. These include, among others, interactions of the measuring instrument with the space charge system, schemes for calibration of instruments, mobility spectrum of the ions making up the space charge and control of the ion density. 


\section{Ion Dens ity Measurements}

\section{A. Introduction}

The continual production of ions by the corona discharge associated with a high voltage dc transmission line results in the presence of space charge which modifies the nominal electric field near the line and causes a vertical current to ground in the vicinity of the line. These ion-related effects are discussed in other sections of this report. Other ion related electrical quantities include the homopolar charge density, the net space charge (which is the algebraic sum of the positive and negative homopolar charge densities), the conductivity, and the ion mobility spectrum. Measurements of these quantities have been of interest to the atmospheric electricity community for many decades and various instruments and measurement techniques are described in the literature [2].

However, in the absence of severe thunderstorms, the electrical environment encountered in atmospheric electricity research is much less hostile than that encountered near a high voltage dc transmission line. As a result, demands are placed on instruments which far exceed those for which they were originally designed. This requires that the operation of the instruments under conditions like those near a line must be characterized. In addition, there is concern about absolute calibration of instruments and assessment of errors associated with various measurement techniques.

Emphasis in the effort described here has been placed on measurements of space charge density and homopolar charge density. Knowledge of conductivity provides a useful correlation between other measurements, but no information 
about quantities of direct interest. Mobility measurements are of concern, but have been deferred to the future.

The measurement of the homopolar charge density in air at atmospheric pressure has traditionally been done using instruments called ion counters. These aspiration-type devices do not actually count ions, but measure charge per unit volume.

Ion counting involves the use of instruments which, in the simplest form, continuously draw ion-laden air from the atmosphere. The ions in the air are subjected to electrical forces in the ion counters which cause them to precipitate out on collecting electrodes. A measurement of the ion current to these electrodes, the air flow through the instrument and certain geometrical characteristics of the instrument allow a calculation of the homopolar charge per unit volume of the air being sampled. By measuring both positive and negative charge densities, an estimate of the net space charge can be obtained by subtraction. If the charge densities are comparable, this subtraction can lead to a large uncertainty because of limited instrumental resolution. A knowledge of the mobility spectrum is also required for complete evaluation of ion counting instruments, since not all ions with mobilities below the critical mobility for a given ion counter will be detected.

Measurements of net space charge have been made for many decades and earlier efforts have been summarized in the literature [8]. Two methods which have been used involve (a) the Faraday cage and (b) the "absolute" or high efficiency particulate air (HEPA) filter. For this application, the Faraday cage is an enclosure made of a metallic mesh immersed in a space containing ions. Assuming the ion density is uniform and the cage is of simple geometry, 
a solution of Poisson's equation for the appropriate geometry yields an expression for the potential at any point inside the cage. Measurement of this potential at one point leads to a value for net space charge.

The absolute filter is an aspiration device in which an air sample is drawn through the filter, which is electrically isolated from ground. The HEPA filter has a very high efficiency ( $>99.7 \%$ ) for removing particles of diameters 0.3 micrometer and larger from the air stream. If these particles carry charge, this charge will be deposited on the filter and by measuring the current to ground, and the air flow through the filter, the net charge per unit volume may be calculated. At first glance, it would appear that the HEPA filter would not be useful for small ions which have diameters substantially less than 0.1 micrometer. However, previous investigators have shown some evidence that a substantial fraction of small ions lose charge in passing through the filter [9].

We have begun to examine systematically both ion counters and net space charge measurement techniques to identify sources of error, to evaluate their usefulness in determining the electrical environment around a dc transmission line and to ascertain if suitable means for calibrating ion measuring instruments can be developed.

B. Approach and Results

\section{B1. Ion Counters}

One type of ion counter, which uses a parallel plate configuration, is indicated schematically in Fig. 15. A series of parallel plates, alternately connected, is placed in an enclosure. Air containing ions is drawn through the assembly and, with one set of plates energized as shown, ions are precipitated 


\section{TO BLOWER}

(a)

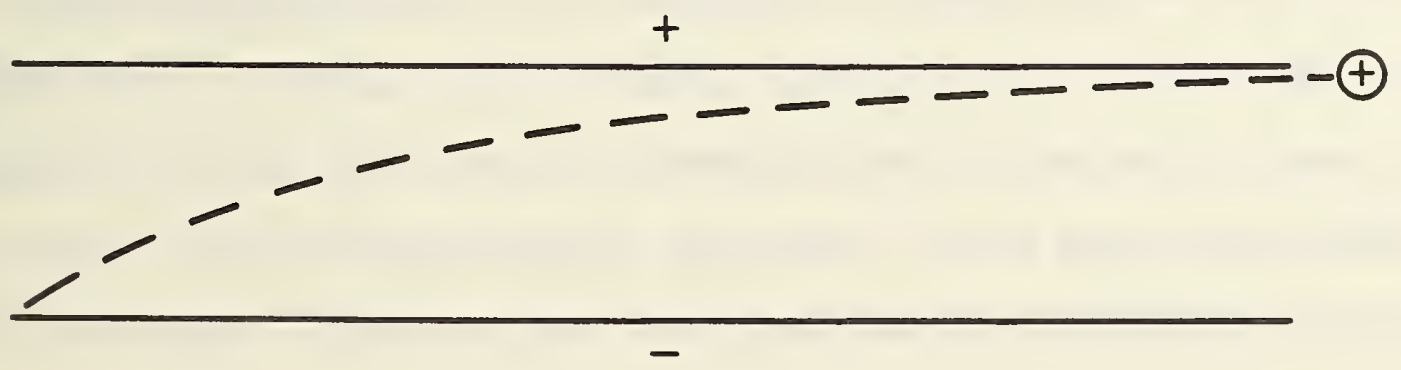

(b)

Figure 15 . (a) Schematic of a parallel plate ion counter.

(b) Trajectory of an ion with critical mobility $K_{c}$. 
onto the collector plates under the action of the electric field. By determining the volumetric air flow rate $\Phi$ and the current to the collector plates, I, the charge per unit volume $\rho$ may be calculated as

$$
\rho=I / \Phi
$$

If the ions are assumed to be singly charged, then an expression for the number density is

$$
N=\frac{\rho}{e}
$$

where $e$ is the electron charge $\left(1.6 \times 10^{-19} \# C\right)$. Not all ions entering the counter will be collected. Consider the trajectory indicated in Fig. 15(b) for an ion which enters at the top of the interplaner space and impinges on the collector plate just at the exit of the counter. For a given geometry, polarizing potential, and volumetric air flow rate, an ion following this trajectory will have what is called the "critical mobility" $K_{c}$. All ions which have mobilities greater than $K_{c}$ will be collected. Some fraction of those ions with mobility less than $K_{c}$ will be collected as well, but the concept of a critical mobility is useful to describe the mobility range of ions collected.

In an extensive discussion of ion counter technology, Tammet [10] summarizes early research on ion counters. It can be shown that the critical mobility for a cylindrically symmetric ion counter is given by

$$
K_{c}=\frac{\varepsilon_{0} \Phi}{C V}
$$


where $\Phi$ is the volumetric air flow rate, $C$ is the capacitance of the active region of the ion counter and $V$ the polarizing voltage. This expression applies to parallel plate counters as well, since they are a limiting case of cylindrical geometry with large radii.

If the current to the collector plates is measured as the polarizing vol tage is increased, an I-V characteristic curve of an ideal counter is obtained as indicated in Fig. 16. Saturation of the current indicates all of the ions entering the counter are being collected.

One serious source of error associated with ion counters is that of ion loss in the inlet structure due to fringing electric fields. Some of the more mobile small ions will be lost to the walls of the instrument and, if the space charge is predominately made up of small ions, large errors can result. If these losses are present, an I-V characteristic curve like that in Fig. 16 will be observed.

The I-V characteristic curves for two different ion counters were measured for different space charge conditions. These measurements were made by connecting to the inlet of the counter a tube $2 \mathrm{~m}$ long and $10 \mathrm{~cm}$ in diameter. An alpha particle source which could be biased relative to ground to provide only positive or only negative ions was placed at the inlet end of the tube. For a fixed bias voltage and constant air flow across the source, a constant average ion density was produced at the inlet of the counter. Current-voltage characteristic curves were obtained for each of the two counters. The results for the first counter are shown in Fig. 17. The saturation is evident for both positive and negative ion measurements. In 


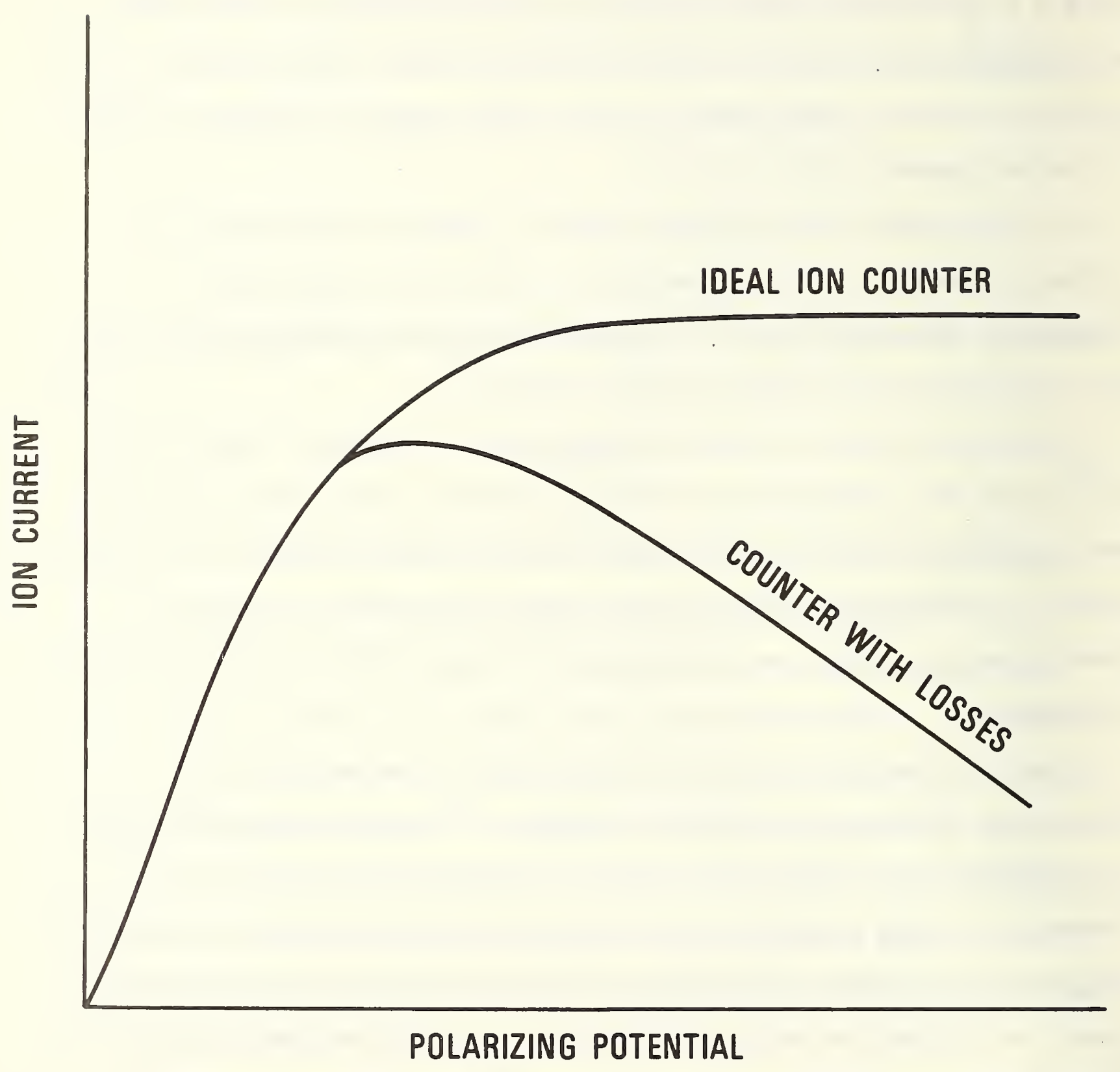

Figure 16. Current-voltage characteristic curves for (a) an ideal ion counter and (b) a counter with losses for a space charge made up of small ions. 


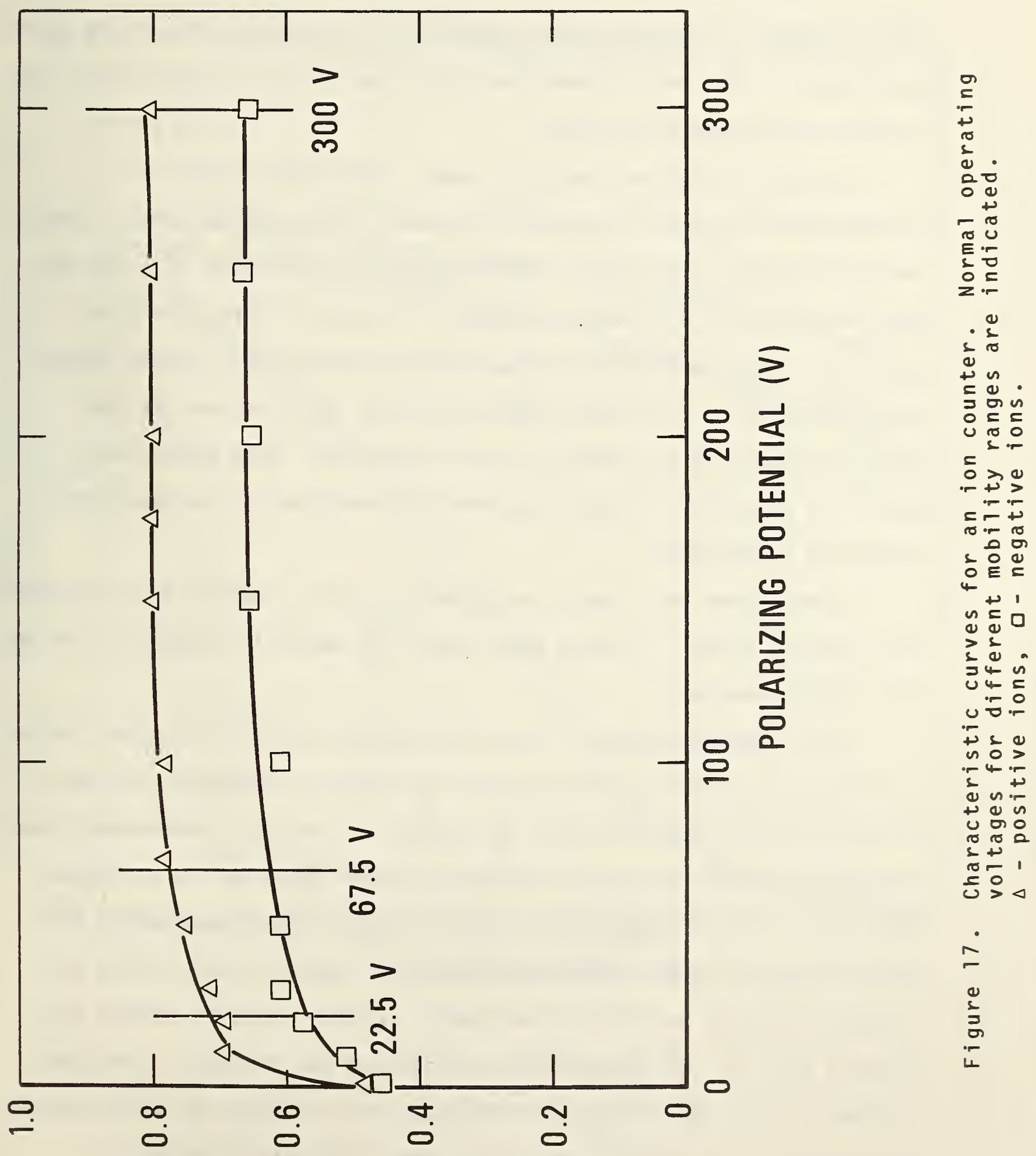

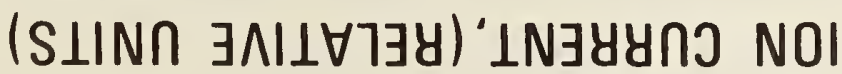


normal operation, fixed polarizing potentials as indicated on the figure would be selected by the user to change the critical mobility. For this counter the volumetric flow rate is constant.

Selection of different mobility ranges in the second counter is accomplished by changing the polarizing potential and, for the largest ions, by reducing the flow rate as well. The characteristic curves for both high and low flow rates for this counter are shown in Fig. 18. There clearly are substantial losses at the inlet associated with this counter. These losses represent errors of 21 percent and 95 percent for positive ions for the operating conditions for smaller critical mobilities. Data points for polarizing voltages below those required for saturation are not shown for clarity of presentation.

In the counter with losses, the polarizing plates protrude slightly beyond the collector plates. It would appear that this design is responsible for the large losses observed.

An ion counter assembly, in which the relative position of polarizing and collector plates can be varied, has been designed and constructed for use in studies of errors associated with ion counters. A series of measurements have been made in which the relative position has been varied and for a constant flow rate, the I-V characteristic curve generated. These measurements have shown clearly the inlet losses associated with counters in which there are fringing fields at the inlet to the counter assembly. Typical results are shown in Fig. 19. The experimental configuration was the same as described previously. Even where the polarizing plates are retracted one plate spacing, there are still significant losses with higher polarizing potentials. 


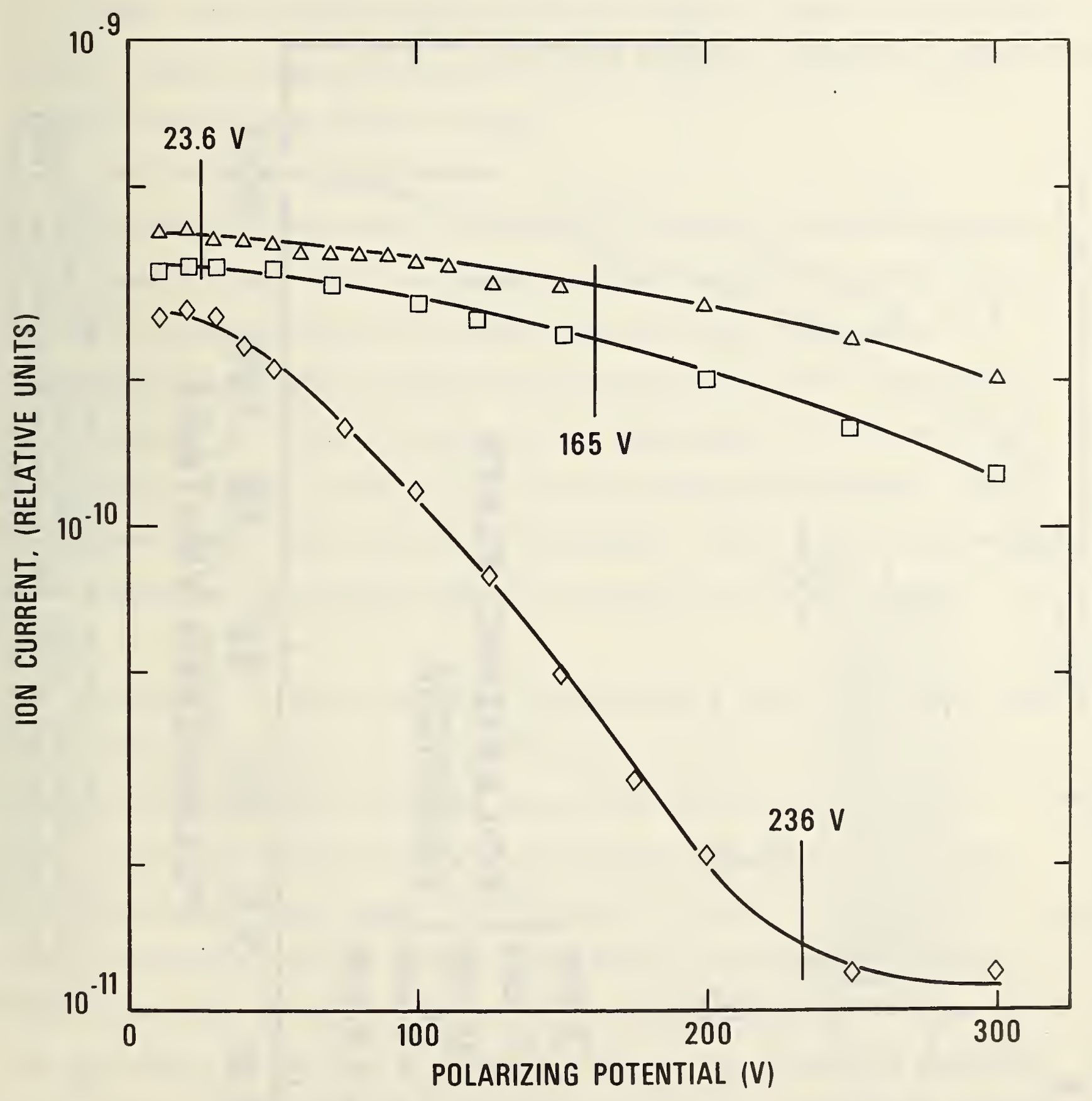

Figure 18. Characteristic curves for an ion counter with losses. Normal operating voltages for different mobility ranges are indicated. $\Delta$ - positive ions, $\square$ - negative ions, $\checkmark$ - positive ions and low volumetric air flow rate. 


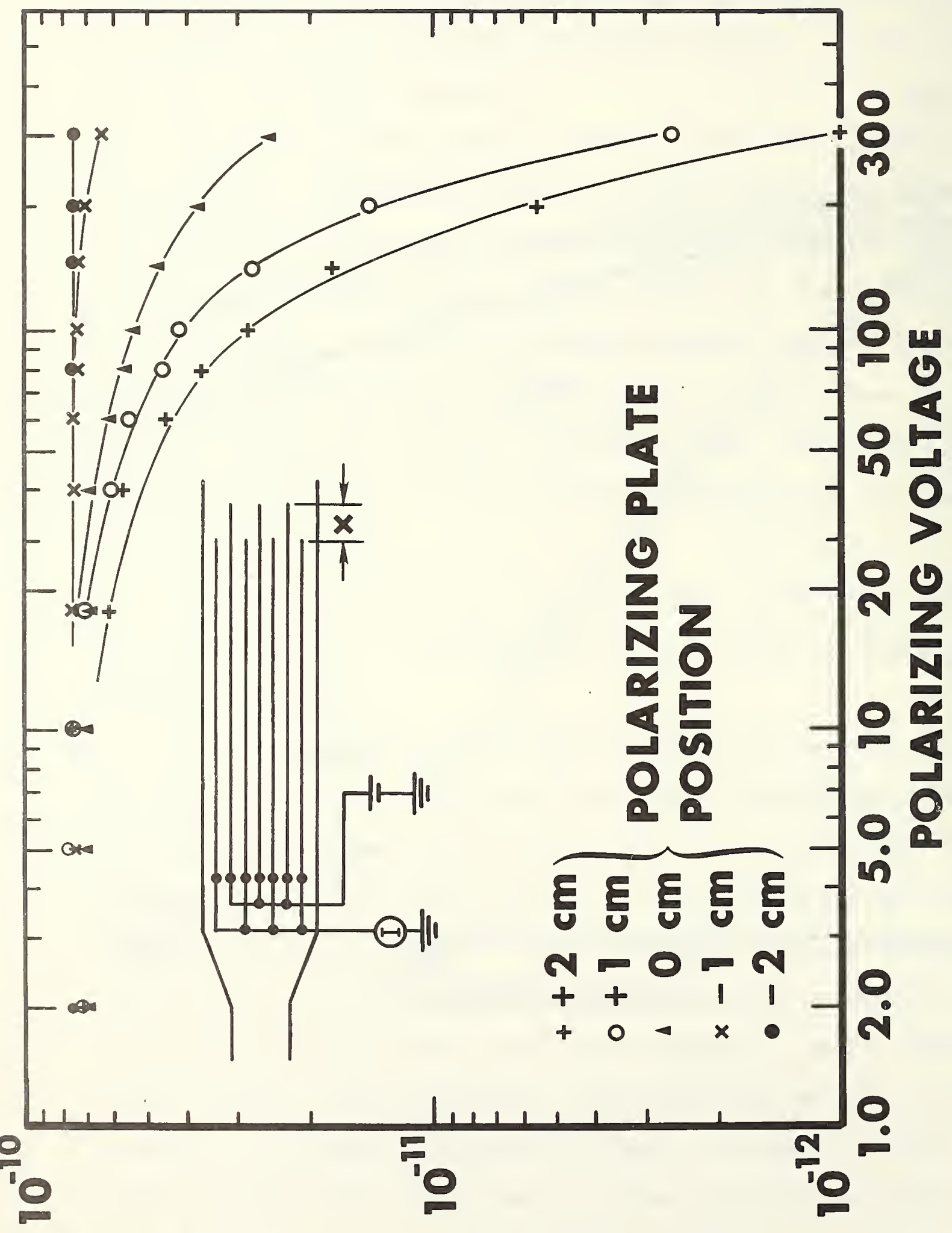

$\stackrel{t}{E} n$

$\stackrel{0}{\oplus}$

ब

U一ㄷㅇ

은

ह $0 \leq u$

는응

N.r.r.r

- $0 \mathrm{~N}+5$

2 . 00

ช \& 200

앙 $\backsim$

$0+$

4 is

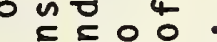

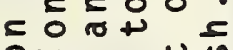

O.m

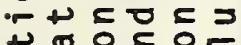

4 工

ᄃ 3 中 24

5 ơ य in

$4-\tau 00=8$

$4-x \cdot \pi$

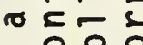

๓ $0000 \%$

ه

ब $0 \times 2+$

$++\infty \times \frac{10}{0}$

ᄃ

बर 0 क

द24 $n$ a

J十 0 in

$u=$ u 0

a $0=+$

$+<\sigma_{\sigma}^{0} \sigma 0^{\circ}$

उ)

안 111

$\geq 0>0 x$

उ०ण $0 \geq 0$

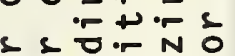

(1) 0 \% $n$.

$+40 \%$

= $-a$.

उ० ơ $\overline{0}$ i

U

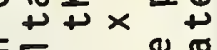

등 4 थ

$\mapsto>0.0+\frac{1}{0}$

o 
Other sources of error such as turbulence in the air stream, space charge effects, losses in inlet ducting and instrument potential relative to space potential have not been studied in detail.

\section{B2. Net Space Charge Measurements}

An absolute filter assembly was constructed for use in laboratory studies and is shown in Figure 20. This assembly has been designed for maximum utility and can be readily adapted for different inlet and outlet connections. A linear scanning and support system has been particularly useful in evaluating the performance of various ion sources in the development of a facility to produce space charge. Volumetric air flow rate through the instrument filter is measured using a calibrated turbine flow meter. The air-handling system has been designed to allow the flow rate to be varied from 2 to 20 liters per second.

The absolute filter has potential usefulness as a device which can be used to calibrate ion counters. If a homopolar space charge is produced, then the hornopolar charge density and the net space charge density are equivalent. A measure of net space charge density yields the absolute charge density, the knowledge of which may be used in calibrating ion counters. The reason that the two measurements are not directly comparable is the following. The ion counter has associated with it some critical mobility and as a result, may or may not collect all the ions in a given air sample. If the mobility spectrum of the ions is known, then the counter can be operated in a mode which insures collection of approximately all the ions. Assuming that this is the case, the absolute filter may be used to calibrate ion counters if the absolute filter is 100 percent effective at removing ions from an air stream. 


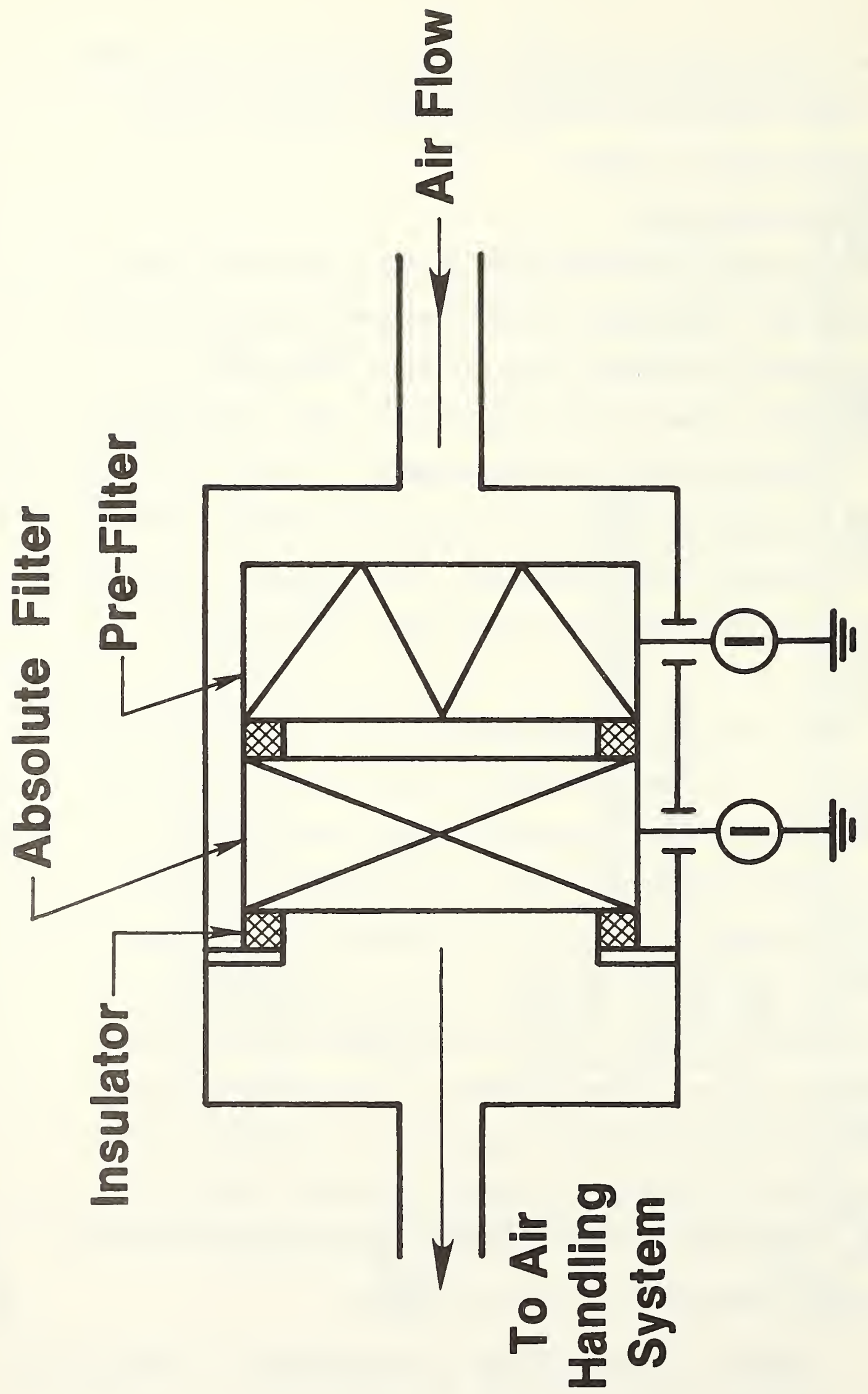


To show this, the absolute filter can be connected in series with an ion counter or conductivity tube. Since only small (molecular) ions would be expected to pass through the filter (based on the particulate trapping efficiency), an ion counter would indicate the charge density in the exhaust air from the absolute filter. By comparing the charge densities indicated by the two instruments, a measure of the efficiency of the absolute filter in removing ions from the air stream can be obtained.

This efficiency must be determined as a function of several variables including flow rate, ion density and ion charge. These studies will use the new space charge facility which is being developed.

In considering methods for obtaining an absolute measure of space charge density, the technique of the Faraday cage cannot be neglected. A Faraday cage for this purpose is an enclosure constructed of open mesh conducting screen. Traditionally, the Faraday cage has been used with a radioactive equilibrated "antenna" to yield a measure of the potential at the geometrical center of a cubic enclosure. In our considerations of Faraday cages, we have obtained solutions to Poisson's equation for both rectangular boxes and right circular cylinders. Figure 21 shows the geometry of these cages and Eqs. 10 and 11 are the formal solutions of Poisson's equation with the assumption that the potential of the boundary is zero. 

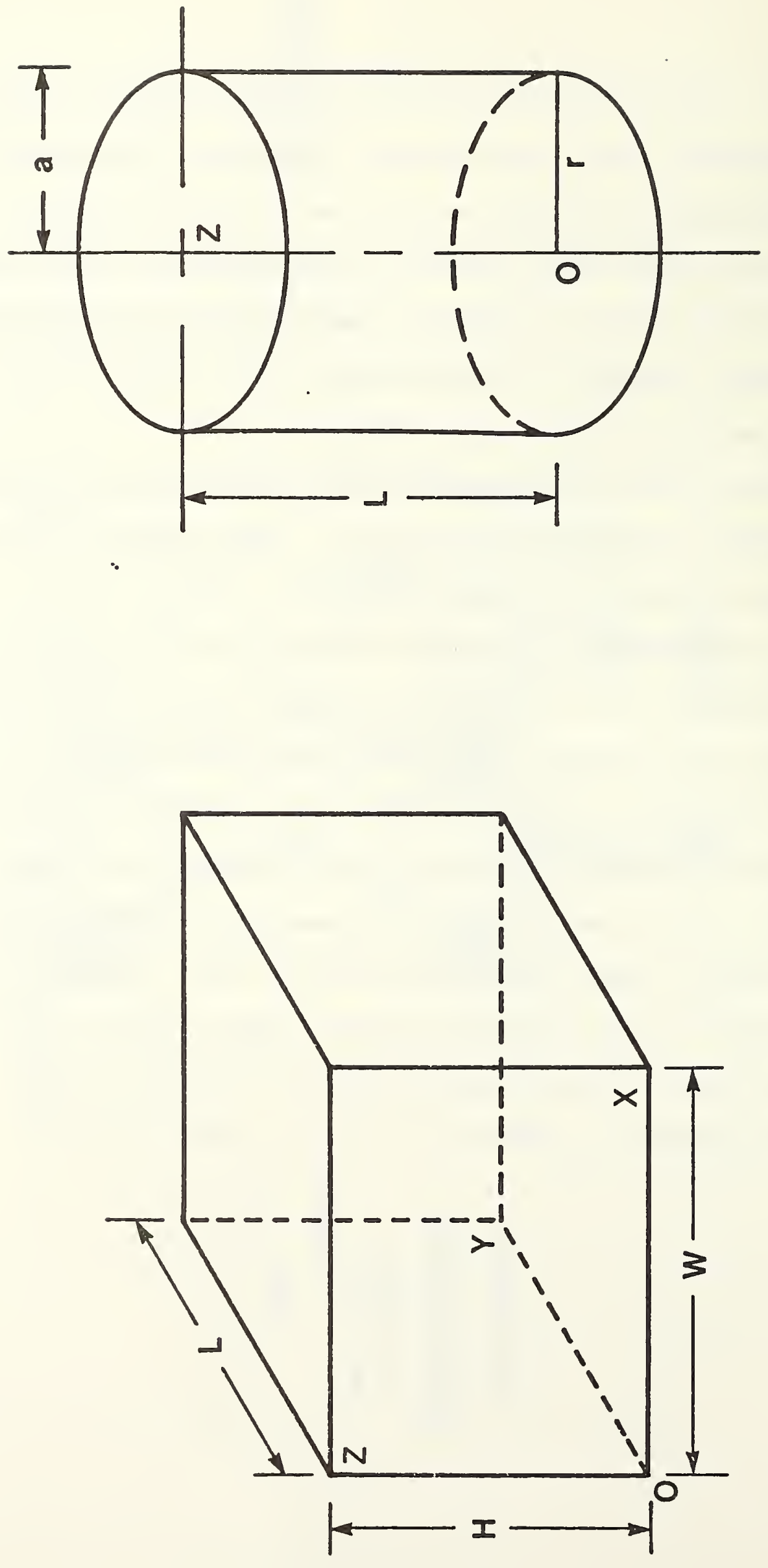

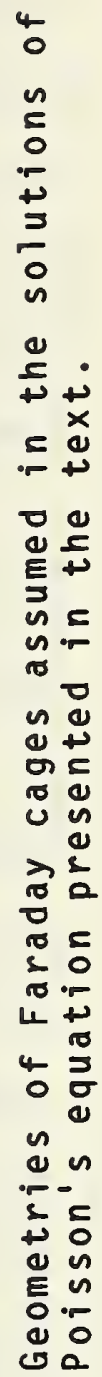

0
0
5
5
0
0
11 
For the rectangular enclosure,

$$
\phi=\frac{64 \rho}{\varepsilon_{0} \pi^{3}} \sum_{\substack{i, j, k \\(\text { odd })}} \frac{\sin \left(\frac{i \pi x}{W}\right) \sin \left(\frac{i \pi y}{L}\right) \sin \left(\frac{k \pi z}{H}\right)}{i \cdot j \cdot k \cdot\left[\left(\frac{i \pi}{W}\right)^{2}+\left(\frac{j \pi}{L}\right)^{2}+\left(\frac{k \pi}{H}\right)^{2}\right]}
$$

The solution for a right circular cylinder is

$$
\phi=\frac{z \rho}{\varepsilon_{0} a} \sum_{n=1}^{\infty} \frac{J_{0}\left(k_{n} r\right)}{k_{n}^{3} J_{1}\left(k_{n} a\right)}\left\{\frac{\left(\cosh \left(k_{n} L\right)-1\right) \sinh \left(k_{n} z\right)}{\sinh \left(k_{n} L\right)}-\left(\cosh \left(k_{n} z\right)-1\right)\right\} .
$$

In each of these, $\rho$ is the net space charge density. In Eq. 11, Jo and $J_{1}$ are Bessel functions of zero and first order, respectively, and $k_{n}$ is determined by the requirement that $J_{0}\left(k_{n} a\right)=0$.

A computer program has been written to evaluate a truncated series of the form given in Eq. 10. Calculations were made for the potential at different locations within the cage. During the course of these calculations, a discrepancy was noted between our results and the widely used calculation of Dodson [11] referred to by Vonnegut and Moore [8] and later reproduced by Chalmers [2]. The potential at the geometrical center of a cube may be written 
in simplified form as

$$
V=\text { const. } \rho L^{2}
$$

where $\rho\left(\frac{c}{m^{3}}\right)$ is the net space charge in the box and $L$ is the length of one side of the box. The constant may be evaluated by summing the relevant parts of Eq. 1. Our solutions gave

$$
\text { const. }=6.37 \times 10^{9}
$$

while the work of Dodson, based on a semi-empirical calculation give a value for the const. $=8 \times 10^{9}$. It should be noted that the present results are in agreement with the extensive calculations of Perper and Penney [12]. The source of this discrepancy is unknown, al though no estimate of errors inherent in Dodson's calculation were given by Vonnegut and Moore.

C. Discussion and Conclusions

The use of ion counters and other space charge density measuring devices to characterize the ion environment under a dc transmission line or in a biological exposure system requires an analysis of errors associated with such measurements and the development of techniques for an absolute calibration of the instruments. The results presented here show the effect of one possible source of error, the loss of ions at the inlet of an ion counter due to fringing fields. These losses can be eliminated by properly designing the ion counter. 
An absolute filter assembly to be used in measuring net space charge has been constructed and will be critically examined to determine the potential for use in an absolute calibration of ion counters. There is good indication in preliminary results that the use of the absolute filter in conjunction with the space charge facility will afford a means of calibrating ion counter devices.

\section{Discussions and Conclusions}

Two facilities, which have been and will be used to evaluate various instruments used to characterize the electrical environment around high voltage dc transmission lines, have been developed. A parallel plate system for producing a field accompanied by space charge in which the total field can be calculated has been constructed and used to compare three different electric field measuring instruments. The results of this work were reported in a separate publication [3].

A specific source of error associated with measurements of vertical current density using Wilson plates has been investigated using the parallel plate system. Lack of coplanarity of the sensing element with the ground plane results in a field enhancement at the surface of the sensor. The enhancement factors for several Wilson plate configurations were determined using ac techniques. Preliminary work using dc field and ions shows a correlation between the ac and dc measurements for all plates except those without a guard ring. These studies will be continued in the coming year.

A second development effort is aimed at producing a region containing a space charge which is spatially uniform and temporally smooth. A low-speed, air-flow facility has been used to investigate various ion source 
configurations. Studies show that suitable space charge can be produced if a uniform air stream is used to transport ions from the source of ionization to the test region. The charge density produced depends on the air speed, corona current and source configuration. The results of these studies were used to design and fabricate a new system which will be evaluated in future work. This source of space charge will be used to study ion measuring instruments.

A study of errors associated with measurement of homopolar space charge density by ion counters which result from fringing fields at the center inlet has been made. Although incomplete, the results of this work indicate that properly designed instrumentation is required to avoid substantial error. Two ion counters have been examined, one of which displayed a large error.

A filter system using a high efficiency particulate air filter was used in measurements of space charge density produced in the low-speed air-flow facility. This system has potential use in providing an absolute calibration technique for ion counters and will be investigated further.

Much has been accomplished toward the objectives stated earlier in this communication. As is frequently the case in a effort such as this, progress in one area depends on results in another so that much of what is reported here represents progress and not final results. The availability of the parallel plate facility and the low-speed air-flow system for producing space charge has provided test systems which will allow us in the future to concentrate more on instrument evaluation and characterization. 


\section{References}

1. IEEE Standard 644, "Recommended Practices for Measurement of Electric and Magnetic Fields from AC Power Lines."

2. J. A. Chalmers, Atmospheric Electricity, 2nd ed. (Pergamon Press, 0xford, $1967)$ p. 185.

3. M. Misakian, "Generation and Measurement of DC Electric Fields with Space Charge," Nat. Bur. Stand. (U.S.), NBSIR 80-2177 (1980).

4. P. Moon and D. E. Spencer, Field Theory for Engineers, (D. Van Nostrand Co., Princeton, N.J., 1961).

5. W. P. Winn and L. G. Yerley, III, "Electric Field Growth in Thunderclouds," Quart. J. R. Met. Soc., 101, 1975, p. 993.

6. E. Weber, Electromagnetic Fields (John Wiley \& Sons, Inc., New York, 1950).

7. W. R. Smythe, Static and Dynamic Electricity, 3rd Ed. (McGraw-Hill Book Co., New York, 1968, p. 131).

8. B. Vonnegut and C. B. Moore, "A Study of Techniques for Measuring the Concentration of Space Charge in the Lower Atmosphere" (final report, Contract AF 19 (604) 1920, 31 Jan 1958).

9. R. B. Bent, "The Testing of Apparatus for Ground Fair-Weather Space-Charge Measurements," Journ. of Atm. and Terr. Physics, 26, 1964, p. 313. 
10. H. F. Tammet, "The Aspiration Method for the Determination of AtmosphericIon Spectra," Scientific Notes of Tartu State University, Issue 195 (translated from Russian), 1970. Available from U.S. Department of Commerce, Clearinghouse for Federal Scientific and Technical Information, No. TT68-50499.

11. W. B. Dodson, "A Discussion of Methods for the Measurement of Space Charge Density" (unpublished report for the Research Laboratory, American Air Filter Company, Inc., Louisville, KY 1950).

12. S. Perper and G. W. Penney, "Electric Potentials and Fields in Rectangular Rooms," ASHRAE Transactions, 76, 1970, p. 87. 
Appendix I - Model Calculations of Perturbations to Ambient Fields Caused by Instrument Support Structure

An exact calculation of the field distortion produced by the introduction of a conducting cylinder of finite length into a uniform electric field is difficult. In the present context interest is in the distortion produced at a considerable distance from it by a long thin cylinder with its axis aligned with the field. An analytical solution valid to a high degree of approximation is feasible for this case if the cylinder is represented by a prolate spheroid.

The field in the vicinity of a spheroid of high permittivity (or conducting) material immersed in a previously uniform field, Eo, in air is given in prolate spheroidal coordinates as:

$$
\begin{aligned}
& E=\left(E_{0} /\left(\sinh ^{2} n+\sin ^{2} \theta\right)^{1 / 2}\right)\left\{-\vec{a}_{n}[\sinh n-\right. \\
& \left.\cosh n_{0} Q_{1}{ }^{\prime}(\cosh n) / Q_{1}\left(\cosh n_{0}\right)\right] \cos \theta+\vec{a}_{\theta}[\cosh n- \\
& \left.\left.\cosh n_{0} Q_{1}(\cosh n) / Q_{1}\left(\cosh n_{0}\right)\right] \sin \theta\right\}
\end{aligned}
$$

where

$\vec{a}_{n}$ is the unit vector normal to the $n=$ constant surfaces;

$\vec{a}_{\theta}$ is the unit vector normal to the $\theta=$ constant surfaces;

$Q_{1}(\mu)$ is a Legendre function of the second kind;

$Q_{1}^{\prime}(\mu)$ is the first derivative of $Q_{1}(\mu)$; and $n_{0}$ is the value of $n$ which defines the spheroid.

The prolate spheroidal coordinates are related to rectangular coordinates by the equations (see also Fig. Al):

$$
\begin{aligned}
& x=a \sinh n \sin \theta \cos \psi \\
& y=a \sinh n \sin \theta \sin \psi \\
& z=a \cosh n \cos \theta .
\end{aligned}
$$

Surfaces of constant $n$ are prolate spheroids defined by the equation: 


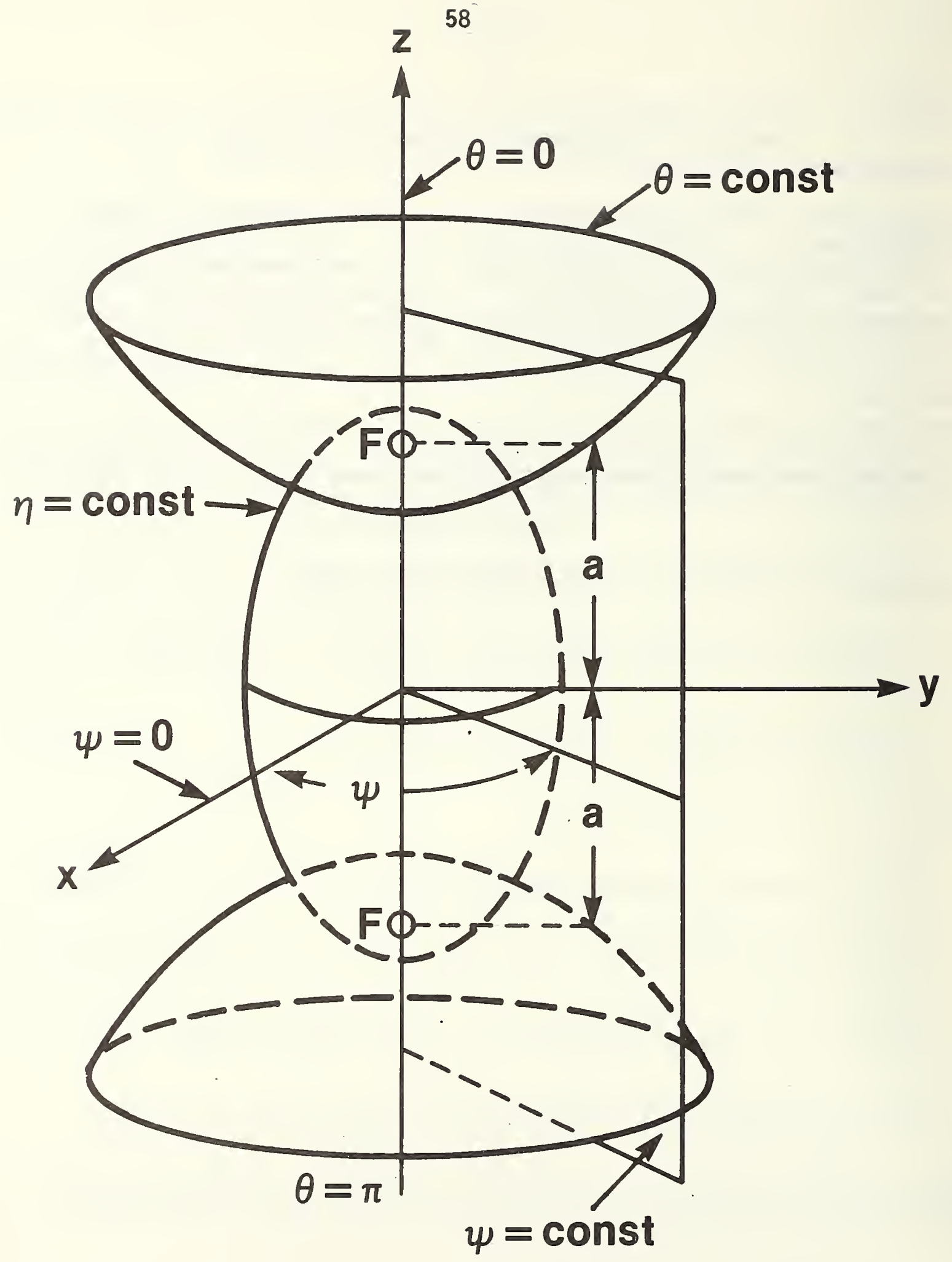

Figure A-1. Prolate spheroidal coordinates. The coordinate surfaces are prolate spheroids, $n=$ const., hyperboloids $\theta=$ const., and meridian planes $\psi=$ const. 


$$
x^{2} / b^{2}+y^{2} / b^{2}+z^{2} / c^{2}=1
$$

where

$$
\begin{aligned}
& b=a \sinh n \text { and } \\
& c=a \cosh n .
\end{aligned}
$$

(Note that $\left.c^{2}-b^{2}=a^{2}\right)$.

With the major axis of the spheroid parallel to the unperturbed field vector the field will be symmetric about the $z$ axis and it is advantageous to introduce the variable, $r$,

$$
r \equiv\left(x^{2}+y^{2}\right)^{1 / 2}=a \sinh n \sin \theta .
$$

Surfaces of constant $n$ then may be written

$$
r^{2} / b^{2}+z^{2} / c^{2}=1
$$

and surfaces of constant $\theta$,

$$
-r^{2} / b^{2}+z^{2} / c^{2}=1 .
$$

In the present application interest is in the field at a considerable distance from the spheroid, where the perturbations resulting from its presence are at most a few percent. Hence only the region where $n$ is large is of interest. In that region

$$
\sinh n \simeq \cosh n \simeq e^{n / 2} .
$$

Now define a new variable, $R$, such that

$$
R \simeq a e^{n / 2} \simeq a \sinh n \simeq a \cosh n \ldots
$$

Then $r$ and $z$ in terms of this new variable become

$$
\begin{aligned}
& r \simeq R \sin \theta \\
& z \simeq R \cos \theta .
\end{aligned}
$$


Also note that $b \simeq c \simeq a e^{n / 2} \simeq R$

$a$, which is small relative to $b$ and $c$, is approximately equal to the semimajor axis of the conducting spheroid.

It is necessary to express the unit vectors $\vec{a}_{\eta}$ and $\vec{a}_{\theta}$ in Eq. I in terms of the unit vectors of the new variables. Note that for the $n=$ constant surface,

$$
\begin{aligned}
& u(r, z)=r^{2} / b^{2}+z^{2} / c^{2} \\
& d u / d r=2 r / b^{2}, \quad d u / d z=2 z / c^{2} .
\end{aligned}
$$

The normal vector is

$$
\vec{n}=(d u / d r) \vec{a}_{r}+(d u / d z) \vec{a}_{z}=\left(2 r / b^{2}\right) \vec{a}_{r}+\left(2 z / c^{2}\right) \vec{a}_{z}
$$

and the unit normal vector is

$$
\begin{aligned}
& \vec{a}_{n}=\left(\left(2 r / b^{2}\right) \vec{a}_{r}+\left(2 z / c^{2}\right) \vec{a}_{z}\right) /\left(\left(2 r / b^{2}\right)^{2}+\left(2 z / c^{2}\right)^{2}\right)^{1 / 2} \\
& =\left(r\left(c^{2} / b^{2}\right) \vec{a}_{r}+z \vec{a}_{z}\right) /\left(\left(r c^{2} / b^{2}\right)^{2}+z^{2}\right)^{1 / 2} .
\end{aligned}
$$

Tangent to the $n=$ constant surface

$$
\begin{aligned}
& d u(r, z)=0=2 r d r / b_{2}+2 z d z / c^{2} \\
& \text { and } \quad d r / d z=-z b^{2} / r c^{2} .
\end{aligned}
$$

Then the tangent vector is

$$
\dot{\theta}=a b^{2} \vec{a}_{r}-r c^{2} \vec{a}_{z}
$$

and the unit tangent vector is 


$$
\begin{aligned}
& \vec{a}_{\theta}=\left(z b^{2} \vec{a}_{r}-r c^{2} \vec{a}_{z}\right) /\left(\left(z b^{2}\right)^{2}+\left(r c^{2}\right)^{2}\right)^{1 / 2} \\
& =\left(z \vec{a}_{r}-\left(r c^{2} / b^{2}\right) \vec{a}_{z}\right) /\left(z^{2}+\left(r c^{2} / b^{2}\right)^{2}\right)^{1 / 2} .
\end{aligned}
$$

Since in the region of concern $b \simeq c \simeq R$ equations $A 6$ and $A 7$ may be written

$$
\begin{aligned}
\vec{a}_{n} & =\left(r \vec{a}_{r}+z \vec{a}_{z}\right) /\left(r^{2}+z^{2}\right)^{1 / 2} \\
& =\left(R \sin \theta \vec{a}_{r}+R \cos \theta \vec{a}_{z}\right) /\left(R^{2}\left(\sin ^{2} \theta+\cos ^{2} \theta\right)\right)^{1 / 2} \\
& =\sin \theta \vec{a}_{r}+\cos \theta \vec{a}_{z} . \\
\vec{a}_{\theta} & =\left(z \vec{a}_{r}-r \vec{a}_{z}\right) /\left(r^{2}+z^{2}\right)^{1 / 2} \\
& =\left(R \cos \theta \vec{a}_{r}-R \sin \theta \vec{a}_{z}\right) /\left(R^{2}\left(\cos ^{2} \theta+\sin ^{2} \theta\right)\right)^{1 / 2} \\
& =\cos \theta \vec{a}_{r}-\sin \theta \vec{a}_{z} .
\end{aligned}
$$

Also in this region the $n=$ constant surfaces are nearly spherical (see Eq. A3). Use of spherical coordinates is then appropriate (see Fig. A2) with the unit vectors $\vec{i}_{R}$ and $\vec{i}_{\theta}$ where

$$
\vec{i}_{R} \approx \vec{a}_{n} \text { and } \vec{i}_{\theta} \simeq \vec{a}_{\theta} \text {. }
$$

Equation $A 1$ involves Legendre functions of the second kind $Q_{0}(\mu)$ (implicitly) and $Q_{j}(\mu)$ and the derivative of $Q_{p}(\mu), Q_{j}{ }^{\prime}(\mu)$. The fact that in the region of concern $\mu$ is very large permits use of approximate values of these functions.

$$
\begin{aligned}
& Q_{0}(\mu)=(1 / 2) \ln ((1+\mu) /(1-\mu)) \\
& \quad=(1 / 2)[1 n(1+\mu)-1 n(\mu-1)] \\
& \simeq(1 / 2)\left[1 / \mu-(1 / 2)(1 / \mu)^{2}+(1 / 3)(1 / \mu)^{3}+\ldots\right) \\
& \left.\quad-\left(-(1 / \mu)-(1 / 2)(1 / \mu)^{2}-(1 / 3)(1 / \mu)^{3}-\ldots\right)\right] .
\end{aligned}
$$




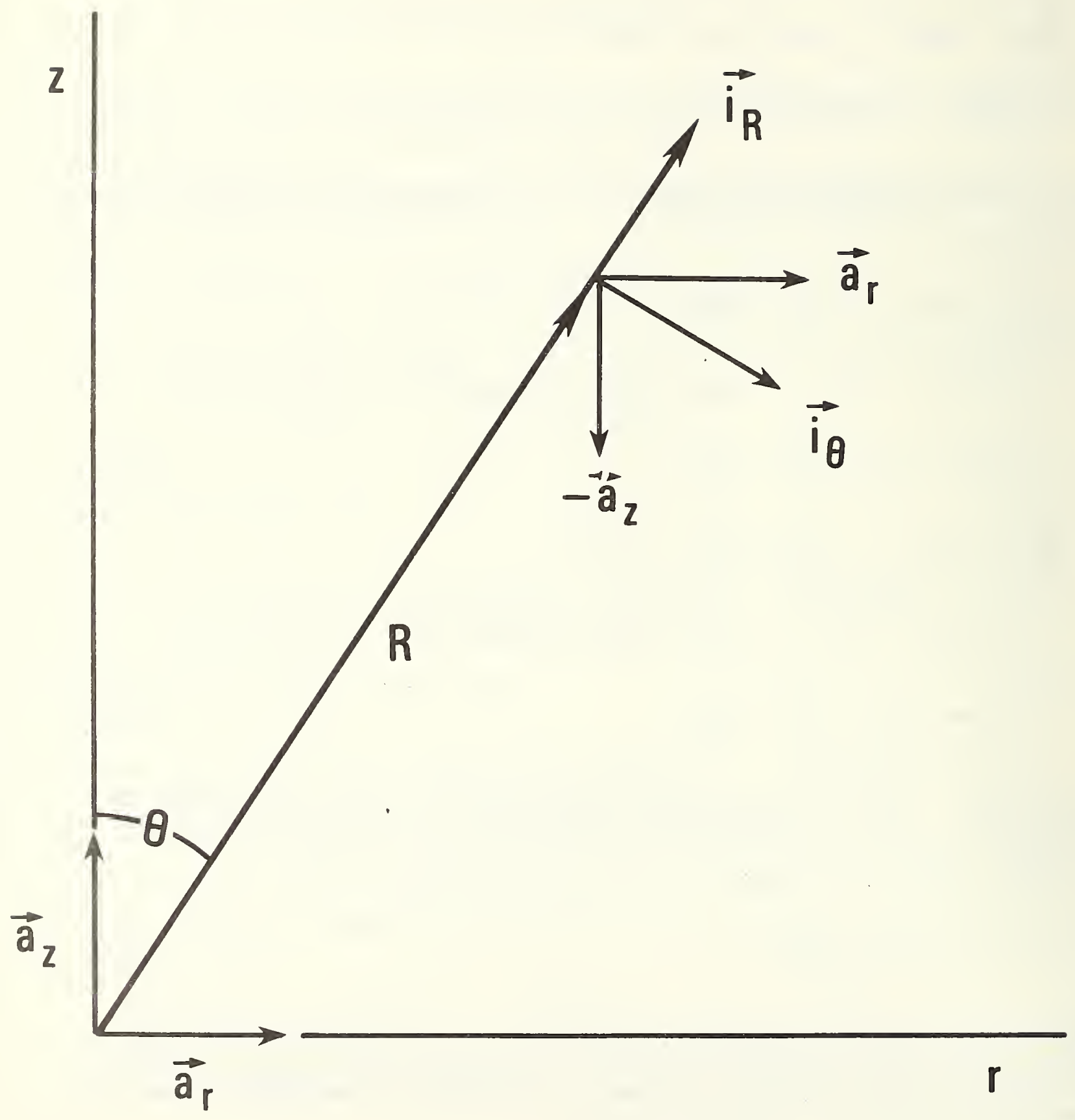

Figure A-2. Representation of various unit vectors used in the development of an expression for the electric field far from a conducting prolate spheroid immersed in a uniform electric field. 


$$
\begin{aligned}
& \text { (Since } \ln (1 \pm(1 / \mu))= \pm 1 / \mu-(1 / 2)(1 / \mu)^{2} \\
& \left.\quad \pm(1 / 3)(1 / \mu)^{3} \ldots\right) \\
& \simeq 1 / \mu \pm(1 / 3)(1 / \mu)^{3}+\ldots
\end{aligned}
$$

Likewise

$$
Q_{1}(\mu)=\mu Q_{0}(\mu)-1
$$

may be written for large values of $\mu$

$$
Q_{1}(\mu) \simeq \mu\left(1 / \mu+(1 / 3)(1 / \mu)^{3}+\ldots\right]-1=\left(1 / 3 \mu^{2}\right)+\ldots
$$

then

$$
d Q_{1}(\mu) / d_{\mu}=Q_{1}{ }^{\prime}(\mu)=-2 / 3 \mu^{3} .
$$

Now substituting from Eqs. A5a, Al0, All, Al2, and $A 13$ into Eq. Al -and noting that

$$
\begin{aligned}
& \left(\sinh ^{2} n+\sin ^{2} \theta\right)^{1 / 2} \\
& \quad=\left[(R / a)^{2}+\sin ^{2} \theta\right]^{1 / 2} \simeq R / a=R /\left(c^{2}-b^{2}\right)^{1 / 2} .
\end{aligned}
$$

Hence in the far field

$$
\begin{aligned}
\vec{E}_{1} & =\left(E_{0}\right) /(R / a)\left\{-\vec{i}_{R}\left[R / a+2 \cosh n_{0}\right.\right. \\
& \left.3(R / a)^{3} Q_{1}\left(\cosh n_{0}\right)\right] \cos \theta \\
+ & \left.\vec{i}_{\theta}\left[R / a-\cosh n_{0} / 3(R / a)^{2} Q_{1}\left(\cosh n_{0}\right)\right] \sin \theta\right\} \\
\simeq & E_{0}\left\{-\vec{i}_{R}\left[1+2 \cosh n_{0} / 3(R / a)^{4} Q_{1}\left(\cosh n_{0}\right)\right] \cos \theta\right. \\
+ & \left.\vec{i}_{\theta}\left[1-\left(\cosh n_{0}\right) / 3(R / a)^{3} Q_{1}\left(\cosh n_{0}\right)\right] \sin \theta\right\},
\end{aligned}
$$


or

$$
\begin{aligned}
& \text { since }-\vec{i}_{R} \cos \theta+\vec{i}_{\theta} \sin \theta=-\vec{i}_{z} \\
& \vec{E}_{1} \simeq-E_{0} \vec{i}_{z}-\vec{i}_{R}\left\{2 E_{0} \cosh n_{0} /\right. \\
& \left.\quad 3(R / a)^{4} Q_{1}\left(\cosh n_{0}\right)\right\} \cos \theta \\
& -\vec{i}_{\theta}\left\{E_{0} \cosh n_{0} / 3(R / a)^{3} Q_{1}\left(\cosh n_{0}\right)\right\} \sin \theta \\
& \simeq-E_{0} \vec{i}_{z}-\left[\left(E_{0} \cosh n_{0}\right) / 3\left(R / a_{0}\right)^{3} Q_{1}\left(\cosh n_{0}\right)\right]\left[\vec{i}_{\theta} \sin \theta\right. \\
& \left.+\vec{i}_{R}(2 \cos \theta) /(R / a)\right] .
\end{aligned}
$$

For a long, slender prolate spheroid $b_{0} / c_{0} \ll<1$

$$
b_{0} / c_{0}=\sinh n_{0} / \cosh n_{0} \simeq n_{0} /\left(1+n_{0}^{2} / 2\right) \simeq n_{0}<1 .
$$

(Using $\cosh n_{0} \simeq 1+n_{0}^{2} / 2$ ) and $\sinh n_{0} \simeq n_{0}$ ).

Note that $a_{0}, b_{0}$, and $c_{0}$ relate to the conducting spheroid.

The Legendre functions for small no or $\mu=\cosh n_{0}$ near 1 may be approximated as

$$
\begin{aligned}
& Q_{0}(\mu) \simeq 1 / 2\left[1 n\left(2+n_{0}^{2} / 2\right)-1 n\left(n_{0}^{2} / 2\right)\right] \simeq 1 / 21 n\left(4 / n_{0}^{2}\right)= \\
& 1 n\left(2 / n_{0}\right) \\
& Q_{1}(\mu) \simeq 1 n\left(2 / n_{0}\right)-1=\ln \left(2 c_{0} / b_{0}\right)-1 \\
& \text { Since } \mu \simeq 1+n_{0}^{2} / 2 .
\end{aligned}
$$

Hence for a long, slender prolate spheroid in the far field

$$
E_{1} \simeq-E_{0}\left\{\vec{i}_{z}+\left[\left(c_{0}^{2}-b_{0}^{2}\right)^{3 / 2} / 3 R^{3}\left(1 n\left(2 c_{0} / b b_{0}\right)-1\right)\right]\right.
$$




$$
\begin{aligned}
& {\left.\left[\vec{i}_{R} 2 \cos \theta\left(c_{0}^{2}-b_{0}^{2}\right) 1 / 2 / R+\vec{i}_{\theta} \sin \theta\right]\right\} } \\
\simeq & -E_{0}\left\{\vec{i}_{z}+\left[c_{0}^{3} / 3 R^{3}\left(1 n\left(2 c_{0} / b\right)-1\right)\right]\right. \\
& {\left.\left[\vec{i}_{R}\left(2 c_{0} \cos \theta / R\right)+\vec{i}_{\theta} \sin \theta\right]\right\} . }
\end{aligned}
$$

Consider the case of a field meter suspended at a height of two meters midway between two 6-cm diameter, 3-meter high poles separated by a distance of 12 meters. Then $b_{0}=0.03, c_{0}=3, R=6.3$, $\theta=1.25$ radians, $\cos \theta=0.32$ and $\sin \theta=0.95$. Substitution of these values in the above equation, recognizing that the horizontal components of the field perturbation produced by the two poles cancel while the verticle components add, gives the following result:

$$
\vec{E}_{1}=-E_{0}(1+0.008) \vec{i}_{z} \text {. }
$$

\section{Appendix References}

A1. P. Moon and D. E. Spencer, Field Theory for Engineers

(D. Van Nostrand Co., Princeton, NJ., p. $252 \mathrm{ff.}$ ) (1961) 
NBS-114AA (REV. 2-8C)

U.S. DEPT. OF COMM.

BIBLIOGRAPHIC DATA

SHEET (See instructions)
1. PUBLICATION OR REPORT NO.

NBSIR 81-2267
2. Performing Organ. Report Nod 3. Publication Date

February 1981

4. TITLE AND SUBTITLE

1980 Annual Report: Electric and Magnetic Field Measurements

5. AUTHOR(S)

R.H. Mcknight, F.R. Kotter, M. Misakian, and P. Ortiz

6. PERFORMING ORGANIZATION (If joint or other than NBS, see instructions)

NATIONAL BUREAU OF STANDARDS

DEPARTMENT OF COMMERCE

WASHINGTON, D.C. 20234

9. SPONSORING ORGANIZATION NAME AND COMPLETE ADDRESS (Street, City, State, ZIP)

7. Contract/Grant No.

8. Type of Report \& Perlod Covered

10. SUPPLEMENTARY NOTES

Document describes a computer program; SF-185, FIPS Software Summary, is attached.

11. ABSTRACT (A 200-word or less factual summary of most significant information. If document includes a significant bibliography or literature survey, mention it here)

The ABS program is concerned with developing methods for evaluating and calibrating instrumentation for use in measuring the electric field and various ion-related electrical quantities in the vicinity of high-voltage direct current (HVDC) transmission lines and in anparatus designed to simulate the transmission line environment.

A parallel plate apparatus designed to produce electric fields in the presence of space charge has been evaluated and used to determine the response of two vibrating plate electric field meters and a field mill.

Errors associated with lack of coplanarity of Hilson plate sensors with the ground plane have been investigated using both ac electric fields and dc electric fields with space charge in a parallel plate apparatus.

A low-speed-air-flow facility has been constructed to produce a volume containing space charge which can be used as a source of ions for evaluatina and comparing instruments desianed to measure various ion related properties.

12. KEY WORDS (Six to twelve entries; alphabetical order; capitalize only proper names; and separate key words by semicolons)

absolute filter; current density; high voltage dc transmission lines;

ion counter; space charge density; Wilson plates

14. NO. OF PRINTED PAGES

65

15. Price

X Order From National Technical Information Service (NTIS), Springfield, VA. 22161 

